\title{
Fat tails in small samples
}

Citation for published version (APA):

Candelon, B., \& Straetmans, S. T. M. (2012). Fat tails in small samples. METEOR, Maastricht University School of Business and Economics. METEOR Research Memorandum No. 014 https://doi.org/10.26481/umamet.2012014

Document status and date:

Published: 01/01/2012

DOI:

10.26481/umamet.2012014

Document Version:

Publisher's PDF, also known as Version of record

\section{Please check the document version of this publication:}

- A submitted manuscript is the version of the article upon submission and before peer-review. There can be important differences between the submitted version and the official published version of record.

People interested in the research are advised to contact the author for the final version of the publication, or visit the DOI to the publisher's website.

- The final author version and the galley proof are versions of the publication after peer review.

- The final published version features the final layout of the paper including the volume, issue and page numbers.

Link to publication

\footnotetext{
General rights rights.

- You may freely distribute the URL identifying the publication in the public portal. please follow below link for the End User Agreement:

www.umlib.nl/taverne-license

Take down policy

If you believe that this document breaches copyright please contact us at:

repository@maastrichtuniversity.nl

providing details and we will investigate your claim.
}

Copyright and moral rights for the publications made accessible in the public portal are retained by the authors and/or other copyright owners and it is a condition of accessing publications that users recognise and abide by the legal requirements associated with these

- Users may download and print one copy of any publication from the public portal for the purpose of private study or research.

- You may not further distribute the material or use it for any profit-making activity or commercial gain

If the publication is distributed under the terms of Article $25 \mathrm{fa}$ of the Dutch Copyright Act, indicated by the "Taverne" license above, 


\section{Maastricht University}

B. Candelon, S. Straetmans

Fat tails in small samples

$\mathrm{RM} / 12 / 014$

\section{METEOR}

Maastricht University School of Business and Economics

Maastricht Research School of Economics

of Technology and Organization

PO. Box 616

$\mathrm{NL}-6200 \mathrm{MD}$ Maastricht

The Netherlands 


\title{
FAT TAILS IN SMALL SAMPLES
}

\begin{abstract}
The tail of financial returns is typically governed by a power law (i.e. "fat tails"). However, the constancy of the so-called tail index $\alpha$ which dictates the tail decay has been hardly investigated. We study the finite sample properties of some recently proposed endogenous tests for structural change in $\alpha$. Given that the finite sample critical values strongly depend on the tail parameters of the return distribution we propose a bootstrap-based version of the structural change test. Our empirical application spans a wide variety of long-term developed and emerging financial asset returns. Somewhat surprisingly, the tail behavior of emerging stock markets is not more strongly inclined to structural change than their developed counterparts. Emerging currencies, on the contrary, are more prone to shifts in the tail behavior than developed currencies. Our results suggest that extreme value theory (EVT) applications in hedging tail risks or in assessing the (changing) propensity to financial crises can assume stationary tail behavior over long time spans provided one considers portfolios that solely consist of stocks or bonds. However, our break results also indicate it is advisable to use shorter estimation windows when applying EVT methods to emerging currency portfolios.
\end{abstract}

\section{INTRODUCTION}

The 1997 Asian crisis, the LTCM debacle or the recent subprime credit crunch have increased the awareness of both academics and practitioners on the importance of accurately assessing the likelihoods of so-called extreme events, i.e. fluctuations in financial markets whose occurrence is relatively rare but can drive banks or institutional investors into overnight financial distress when they strike. However, the academic interest into large tail events is far from new (for an early reference see e.g. Mandelbrot, 1963). He was one of the first to acknowledge that overnight financial market turbulence cannot be captured by the normal distribution function. More specifically, tail probabilities seem to exhibit a polynomial tail decay ("heavy" tails) in contrast to the exponential tail decays of so-called "thin-tailed" models like the normal df. This "heavy tail" characteristic is observed for

Key words and phrases. Tail Index; Extreme Value Analysis; Endogenous stability test; Finite sample properties; Bootstrap.

JEL classification: F31, G15, G19, C49. 
most financial asset classes. Numerous empirical studies have focused on identifying the degree of probability mass in the tail by estimating the so called tail index $\alpha .{ }^{1}$ The integer part of this parameter reflects the number of bounded statistical moments of the corresponding unconditional distribution function (df).

Much less attention has been devoted to the causes and consequences of a non-constant tail index. Conditional volatility models like the GARCH-type class reconcile a stationary unconditional df (constant $\alpha$ ) with clusters of high and low volatility in the conditional df. However, the question arises whether it is realistic to assume that the tail of the unconditional df (and thus measures of long-term risk like unconditional quantiles) remains invariant over long time periods. In other words: can highly volatile periods like the 2007-2010 financial turmoil and periods of market quiescence both be explained by a single unconditional df? Potential causes of shifts in $\alpha$ may include structural changes like e.g. changing trading systems, financial regulatory reform and financial liberalization or changes in the political environment. Moreover, there seems to be a consensus amongst economists that these structural changes are more frequently happening in emerging economies. Our empirical application will therefore distinguish between developed and emerging return tails in order to evaluate whether emerging return tails are relatively more prone to structural shifts in $\alpha$.

Testing for structural change in the tail behavior of the unconditional distribution is relevant from both a statistical and economic perspective. First, whether Extreme Value Theory (EVT) or e.g. the cited GARCH models are applicable depends on the stationarity assumption for the unconditional tail. Also, a non-constant $\alpha$ implies a violation of covariance stationarity which complicates standard statistical inference based on regression analysis. ${ }^{2}$ From an economic perspective, quantifying the correct level of the tail index is relevant for risk managers as it constitutes a necessary ingredient for calculating the

\footnotetext{
${ }^{1}$ Jansen and de Vries (1991), Longin (1996), Lux (1996) and Hartmann et al. (2004) investigated the probability mass in the tails of stock market returns; whereas fat tails in foreign exchange returns have been considered, inter alia, by Koedijk et al. (1990, 1992), Hols and de Vries (1991) and Hartmann et al. (2003). Bond extremes have been rather neglected in the empirical literature; De Haan et al. (1994) and Hartmann et al. (2004) constitute notable exceptions.

${ }^{2} \mathrm{~A}$ popular example constitutes the student-t distribution with $v=\alpha$ degrees of freedom. It is well known that the unconditional variance depends on the tail index: $\sigma^{2}=\alpha /(\alpha-2)$. Thus, time variation in the tail index necessarily transfers into the unconditional variance.
} 
unconditional Value-at-Risk (VaR) very far into the distributional tail, i.e. so-called "tail risk". Indeed, whereas regulatory instances require the financial industry to report and backtest $5 \%$ and $1 \% \mathrm{VaR}$, these events hardly represent extreme events that can trigger financial companies into overnight financial distress. Instead, evaluating downside risk much further into the tail represents useful additional information to e.g. traditional stress testing approaches. Other EVT applications in portfolio selection and risk management include safety first portfolio selection for pension funds (see e.g. Jansen et al., 2000) or the assessment of trading limits for unhedged forex positions in commercial banks (see Danielsson and de Vries, 1997). If the actual value of $\alpha$ in these exercises is incorrectly assessed by e.g. the presence of structural breaks, unconditional VaR quantiles will most probably be biased which in turn may erode the effectiveness of financial risk management and the proper monitoring of overall financial stability (e.g. wrong allocation of risky investments in pension fund portfolios, wrong trading limits for forex traders within banks etc.).

The scant empirical literature on the constancy issue mainly focuses on testing for a single known (i.e. exogenously selected) breakpoint in $\alpha .^{3}$ To the best of our knowledge, Quintos et al. (2001) constitutes the only stability study on detecting single breakpoints in $\alpha$ that also proposes estimators for break dates in $\alpha .{ }^{4}$ Our study extends and refines the previous breakpoint analyses in several directions. First, we select the number of extreme returns to estimate $\alpha$ by minimizing its Asymptotic Mean Squared Error (AMSE) instead of conditioning on a fixed fraction of the total sample. The former approach constitutes common practice in EVT whereas it can be argued that taking a fixed percentage of extremes leads to a degenerate asymptotic limiting df for

\footnotetext{
${ }^{3}$ The scant breakpoint literature includes Phillips and Loretan (1990); Koedijk et al. (1990, 1992); Jansen and de Vries (1991); Pagan and Schwert (1990) and Straetmans et al. (2008). Tests for structural change in $\alpha$ have to be distinguished from cross sectional equality tests (see e.g. Koedijk et al., 1990, on exchange rates or Jondeau and Rockinger, 2003, on stock markets) or asymmetry tests between left and right tails of the same series (see e.g. Jansen and de Vries, 1991, or Lux, 1996, on stock index tail asymmetries).

${ }^{4}$ Werner and Upper (2002), Galbraith and Zernov (2004) and Candelon and Straetmans (2006) already applied the Quintos et al. (2001) methodology to test for tail stability in bund Future returns, US stock market returns and Asian currency returns, respectively. However, they all applied the Quintos et al. asymptotic critical values. We argue in this paper that these critical values do no take into account the bias in the Hill estimator and lead to overrejection of the null hypothesis of tail index constancy.
} 
the $\alpha$-estimator and accompanying stability tests. Second, our simulation study of the stability tests' finite sample properties is much more general than previous studies because we also use data Generating processes that consider higher order tail behavior or empirical stylized facts like e.g. volatility clustering in returns. Last but not least, we apply stability tests to a large cross section of assets and asset classes whereas previous studies typically only focus on a limited number of assets within the same asset class. We also distinguish between developed market financial assets and emerging market financial assets in order to judge whether the latter are more prone to shifts in the tail behavior.

Anticipating our results, we find that size, (size-corrected) power and the ability to detect breaks in finite samples vary considerably with the assumed data generating process (DGP). That is the reason why we propose to bootstrap the critical values in empirical applications for each data set separately. Moreover, the outcomes of our experiments on size-corrected power and the ability to detect breaks suggest that a "recursive" version of the stability test is to be preferred provided the sample is sufficiently large $(n \geq 2,000)$. Applying a bootstrap-based version of this test to a large cross section of assets and asset classes, we mainly detect breaks in the tail behavior of emerging currencies.

The rest of the paper is organized as follows. Section 2 provides a refresher on the statistical theory of heavy tails and accompanying endogenous stability tests. Section 3 contains an elaborate Monte-Carlo investigation of the endogenous breakpoint tests' size, power and break date ability. Section 4 provides an extensive empirical investigation on the tail stability of a variety of developed and emerging asset tails. Section 5 contains concluding remarks.

\section{Testing structural Change in tail Behavior: theory}

We start with a short digression on the theory and estimation of the index of regular variation followed by a short discussion of some temporal stability tests for the tail index $\alpha$.

2.1. Regular variation. We start from the empirical stylized fact that sharp fluctuations in financial market prices exhibit fat tails, see e.g. Mandelbrot (1963) for an early reference or the more recent monograph by Embrechts et al. (1997). Without loss of generality, the presented estimation and testing procedures are expressed in terms of the right tail, i.e. the survivor function $P\{X \geq x\}:=1-F(x)$. Our empirical investigation will focus on sharp drops in the prices of risky securities. This requires taking the negative of a return series prior to 
applying the sketched framework. Under fairly general conditions the survivor function of heavy tailed (or "regularly varying") distributions can be approximated by the second order Taylor expansion for large $x$ :

$$
1-F(x)=a x^{-\alpha}\left(1+b x^{-\beta}+o\left(x^{-\beta}\right)\right),
$$

with $a>0, \alpha>0, b \in \Re, \beta>0$, see e.g. de Haan and Stadmüller (1996). The parameters $\beta$ and $b$ that govern the second order behavior in (2.1) reflect the deviation from pure Pareto behavior in the tail. Notice that if we talk about the "second order parameter" of a fat tailed or regularly varying process later on in the paper, we always refer to the ratio $\rho=-\beta / \alpha$. The case $\beta=\rho=0$ corresponds to the expansion $P\{X \geq x\} \simeq a x^{-\alpha}[1+b \ln x]$. The tail specializes to an exact Pareto when $b=0$.

The regular variation property implies that the (appropriately scaled) upper extremal returns lie in the (maximum) domain of attraction of the Type-II extreme value ("Frechet") distribution. The tail index $\alpha$ reflects the speed at which the tail probability in (2.1) decays if $x$ is increased. Clearly, the lower $\alpha$ the slower the probability decay and the higher the probability mass in the tail of $X$, ceteris paribus the level of $x$. The regular variation property, inter alia, implies that all distributional moments higher than $\alpha$, i.e. $E\left[X^{r}\right], r>\alpha$, are unbounded, signifying "fat tails". Regularly varying probability distributions include the Student-t, symmetric stable, Burr, and Frechet df as well as the GARCH class of conditional volatility models. ${ }^{5}$ As for the tail of the standard normal distribution, a popular tail approximation expresses the survivor function $1-\Phi(\cdot)$ in terms of the density $\phi(x)$ :

$$
\begin{aligned}
1-\Phi(x) & \simeq \frac{\phi(x)}{x}, \quad x \quad \text { large } \\
& =(2 \pi x)^{-1} \exp \left(-\frac{1}{2} x^{2}\right),
\end{aligned}
$$

which clearly describes an exponentially declining tail, see Feller (1971a, p. 175). Distributions with this type of tail decay are classified as "thin tailed" because the tail probability $1-\Phi(x)$ declines much faster to zero as in (2.1); but these distributions possess all moments, and hence do not capture what is typically observed in financial data.

\footnotetext{
${ }^{5}$ Hall (1982) imposes the more stringent condition $\alpha=\beta$ on the tail expansion. This covers certain distributions like the stable laws and the type II extreme value distribution (Frechet); but it does not apply to e.g. the Student-t or the Burr df. For the Student-t df the tail expansion (2.1) holds, though, with $\alpha$ equal to the degrees of freedom $(\alpha=v)$ and $\beta=2$. As for the Burr df, the 2 nd order parameter can be freely chosen. The value of $\beta$ is unknown for the GARCH class.
} 
The focus of the paper will be on the finite sample properties of (single break) temporal stability tests for $\alpha$-estimators. The investigated test statistics use Hill's (1975) estimator for $\alpha$ as an input. Let $X_{1, n} \leq X_{2, n} \leq \cdots \leq X_{n, n}$ represent the ascending order statistics that correspond with the returns series $X$ for a sample of size $n$. Then Hill's estimator boils down to:

$$
\widehat{\alpha}=\left(\frac{1}{m} \sum_{j=0}^{m-1} \ln \left(\frac{X_{n-j, n}}{X_{n-m, n}}\right)\right)^{-1},
$$

such that $\widehat{\alpha}>0$, with $m$ the number of highest order statistics used in estimation. The convergence in distribution of the Hill statistic critically depends on the rate at which the nuisance parameter $m$ grows with the total sample size $n$. The main convergence in distribution result for $\widehat{\alpha}$ is summarized in the following Theorem:

Theorem 1. (Asymptotic normality) Assume that $1-F(x)$ obeys (2.1). If $m, n \longrightarrow \infty$ we distinguish two cases:

(A) If $m=o\left(n^{2 \beta /(2 \beta+\alpha)}\right)$ then $\sqrt{m}(\widehat{\alpha}-\alpha) \stackrel{d}{\longrightarrow} N\left(0, \eta \alpha^{2}\right)$.

(B) If $m=c n^{2 \beta /(2 \beta+\alpha)}$ then $\sqrt{m}(\widehat{\alpha}-\alpha) \stackrel{d}{\longrightarrow} N\left(\varphi \alpha, \eta \alpha^{2}\right)$ for strictly positive and finite $c=\left(\frac{a^{2 \beta / \alpha}(\alpha+\beta)^{2} \alpha}{2 b^{2} \beta^{3}}\right)^{\frac{\alpha}{(2 \beta+\alpha)}}$ and $\varphi=\operatorname{sign}(b)(2 \beta / \alpha)^{-1 / 2}$.

see e.g. Hall (1982) and Haeusler and Teugels (1985) for the i.i.d. case $(\eta=1)$. More recently, convergence in distribution for the Hill statistic has also been established in the presence of temporal dependencies $(\eta \neq 1){ }^{6}$

Loosely speaking, Theorem 1 implies that proper convergence in distribution requires $m$ to rise with $n$ at a "sufficiently slow" pace, i.e., $m, n \rightarrow \infty$ but $m / n \rightarrow 0$. This, however, does not hold when selecting a fixed fraction of extremes $\kappa=m / n$. Previous studies argued that this simple rule-of-thumb performs well in finite samples but its lack of asymptotic justification constitutes a fundamental problem (see e.g. Dumouchel, 1983). We will therefore abstain from using this criterion.

Condition (B) of the convergence Theorem provides a natural alternative towards selecting the nuisance parameter because it can be

\footnotetext{
${ }^{6}$ Hsing (1991) derives consistency and asymptotic normality under serially dependent data. Quintos et al. (2001) extend this result to stationary GARCH processes with conditionally normal innovations. Finally, Drees (2002) derives convergence in distribution for stationary time series processes exhibiting general forms of linear and nonlinear dependence. All these studies conclude that the asymptotic variance for dependent data differs from the i.d.d. variance $\alpha^{2}$. This explains the $\eta$-factor in Theorem 1.
} 
easily shown that the expression for the nuisance parameter $m$ under (B) minimizes the Asymptotic Mean Squared Error (AMSE) for $\widehat{\alpha}$ (see e.g. Danielsson and de Vries, 1997). The AMSE minimization principle is exploited in virtually all empirical EVT studies and we will therefore use this criterion in the rest of the paper. ${ }^{7}$ Theorem 1 also shows that the AMSE criterion induces an asymptotic bias in the Hill statistic, i.e., $E(\widehat{\alpha}-\alpha) \sim m^{-1 / 2} \varphi \alpha$. We will thoroughly document the finite sample consequences of this bias effect on the accompanying stability tests in the Monte Carlo simulation Section 3. For more elaborate expositions on extreme value theory and estimation, see e.g. the monographs by Leadbetter et al. (1983) or Embrechts et al. (1997).

2.2. Structural change in tail behavior. The main goals of the paper are to investigate the finite sample properties of a trio of (single break) stability tests for $\widehat{\alpha}$ introduced earlier by Quintos et al. (2001) and to apply it to detect single breaks in a large set of assets and asset classes from developed and emerging markets. The stability tests differ in the way subsamples are constructed for the Hill estimates. We let $t$ denote the endpoint of a subsample of size $w_{t}<n$. For example, for the full sample one can say that $w_{t}=w_{n}=n$ with $n$ the total sample size. The recursive estimator is defined on subsamples $[1 ; t] \subset[1 ; n]$ as follows:

$$
\widehat{\alpha}_{t}=\left(\frac{1}{m_{t}} \sum_{j=0}^{m_{t}-1} \ln \left(\frac{X_{t-j, t}}{X_{t-m_{t}, t}}\right)\right)^{-1},
$$

with $m_{t}=c t^{\frac{2 \beta}{(2 \beta+\alpha)}}$. The rolling estimator is conditioned on a fixed subsample size $w^{*}<n$. The tail index is estimated by rolling over the subsample, i.e. shifting the subsample through the full sample by eliminating past observations and adding future observations whilst keeping the subsample size constant at $w^{*}$ :

$$
\widehat{\alpha}_{t}^{*}=\left(\frac{1}{m_{w^{*}}} \sum_{j=0}^{m_{w^{*}}-1} \ln \left(\frac{X_{w^{*}-j, w^{*}}}{X_{w^{*}-m_{w^{*}}, w^{*}}}\right)\right)^{-1}
$$

\footnotetext{
${ }^{7}$ In principle, condition (A) could also be used as a selection criterion. For example, choose $\delta>0$ in $m^{*}=c n^{\frac{2 \beta}{(2 \beta+\alpha)}}-\delta$. However, although this criterion guarantees asymptotic unbiasedness, finite sample bias still exists. Moreover, the small sample standard deviation increases with $\delta$. If one cares more about bias than variance, (A) may be an interesting criterion for selecting $m$. In practice, however, researchers typically penalize bias and variance equally and prefer to trade-off bias and variance such as under condition (B). Finally, it is unclear how to choose $\delta$.
} 
with $m_{w^{*}}=c\left(w^{*}\right)^{\frac{2 \beta}{(2 \beta+\alpha)}}$. Finally, the total sample can be partitioned in recursive subsamples $[1 ; t]$ and $[t+1 ; n]$ where $t$ - reflecting the potential break - is shifted through the full sample. Subsample Hill statistics (recursive estimators) can be calculated on both subsamples using (2.3). The recursive Hill estimators for the first and the second recursive subsamples are denoted by $\widehat{\alpha}_{1 t}$ and $\widehat{\alpha}_{2 t}$, respectively. The latter estimator is also sometimes called the "reverse" recursive estimator because it requires (2.3) to be calculated in reverse calendar time. ${ }^{8}$

The three (recursive, rolling and sequential) tests can now be constructed using the sequences:

$$
\begin{aligned}
Y_{n}^{2}(r) & =\left(\frac{t m_{t}}{n}\right)\left(\frac{\widehat{\alpha}_{t}}{\widehat{\alpha}_{n}}-1\right)^{2}, \\
V_{n}^{2}(r) & =\left(\frac{w^{*} m_{w^{*}}}{n}\right)\left(\frac{\widehat{\alpha}_{t}^{*}}{\widehat{\alpha}_{n}}-1\right)^{2}, \\
Z_{n}^{2}(r) & =\left(\frac{t m_{t}}{n}\right)\left(\frac{\widehat{\alpha}_{t}}{\widehat{\alpha}_{2 t}}-1\right)^{2},
\end{aligned}
$$

with $r=t / n$ representing a fraction of the full sample. Expressions (2.5) and (2.6) reflect the fluctuations in the Hill statistic's recursive and rolling values relative to their full sample counterpart $\widehat{\alpha}_{n}$ whereas the sequential test uses (2.7) to compare the fluctuations of the recursive with the reverse recursive estimator.

The null hypothesis of a time invariant tail index $\alpha$ boils down to:

$$
H_{0}: \alpha_{[n r]}=\alpha, \quad \forall r \in R_{\varepsilon}=[\varepsilon ; 1-\varepsilon] \subset[0 ; 1],
$$

with $[n r]$ representing the integer value of $n r$. One would like to test against the two-sided alternative hypothesis $H_{A}: \alpha_{[n r]} \neq \alpha$. For sake of convenience the above test is calculated over compact subsets of $[0 ; 1]$, i.e., $t$ equals the integer part of $n r$ for $r \in R_{\varepsilon}=[\varepsilon ; 1-\varepsilon]$ and for small $\varepsilon>0$. Sets like $R_{\varepsilon}$ are often used in the construction of parameter constancy tests (see e.g. Andrews, 1993). ${ }^{9}$ Conform with Quandt's

\footnotetext{
${ }^{8}$ The recursive Hill statistic $\widehat{\alpha}_{2 t}$ is calculated using eq. (2.3) on the subsample $[n ; t+1]$ where the chronology of the observations has been inverted, i.e. the most recent observations are put at the beginning of the subsample.

${ }^{9}$ The restricted choice of $r$ implies that $\varepsilon n \leq t \leq(1-\varepsilon) n$. When the lower bound is violated the recursive estimates may become too unstable and inefficient because of too small subsample sizes. On the other hand, the test will never find a break for $t$ equal or very close to $n$, because the test value (2.5) is close to zero in that latter case. Thus, for computational efficieny one might stop calculating the tests beyond the upper bound of $(1-\varepsilon) n<n$. Conform with Andrews (1993), we set $\varepsilon=0.15$.
} 
(1960) seminal work on endogenous breakpoint determination in linear time series models, the candidate break date $r$ is selected where the sequences (2.5), (2.6) and (2.7) reach their supremum. This renders the most likely time point for the constancy hypothesis to be violated.

\section{Monte Carlo experiments}

We investigate the finite sample behavior of the recursive, rolling and sequential test for a variety of stochastic models - both for the conditional and the unconditional df - used in the modelling of financial time series. Each model exhibits regularly varying tails and obeys the asymptotic second order expansion (2.1). The number of upper order extremes for the Hill statistic minimizes the Asymptotic Mean Squared Error of the Hill estimator. We calculate finite sample critical values and size-corrected finite sample power against a variety of realistic break scenarios as alternative hypotheses. Last but not least, we report simulated break estimates averaged over the statistically significant breaks at the $95 \%$ significance level.

A short description of the main data generating processes is provided in Subsection 3.1. The analytic derivation of the nuisance parameters for these Data Generating Processes (DGP's) is discussed in Subsection 3.2. Finite sample critical values, size-corrected power properties and the ability to date breaks are evaluated in Subsection 3.3.

3.1. Data generating processes. We choose a variety of heavy tailed DGP's and accompanying parameter values $(a, b, \alpha, \beta)$ that all obey the asymptotic expansion (2.1). Monte Carlo simulations are based on the symmetric stable df, Student-t, Burr, i.e. $P\{X>x\}=\left(1+x^{\beta}\right)^{-\alpha / \beta}$, $\mathrm{AR}(1)$ with stable innovations, $\operatorname{GARCH}(1,1)$ with conditionally normal errors and a Stochastic Volatility model. ${ }^{10}$ Thus, we distinguish between i.i.d. draws and dependent draws. The tail index of the Student-t and Burr is varied between 2 and 4 which is in line with all previous empirical research on heavy tails in financial markets. ${ }^{11}$

Previous studies, including the Quintos et al. (2001) paper, only studied the finite sample behavior of stability tests for $\alpha$ under the class of stable distribution functions (dfs). However, the symmetric stable model has some severe drawbacks as a device for modelling financial returns. First, the property that sums of stable dfs remain

\footnotetext{
${ }^{10}$ Symmetric stable draws are generated using the algorithm by Samorodnitsky and Taqqu (1994).

${ }^{11}$ The Burr distribution is admittedly also not a realistic model for financial return modelling but it enables one to investigate the effects of changing the second order parameter $\beta$ while keeping the tail index constant.
} 
stable distributed after appropriate scaling (additivity property) seems overly restrictive for real-life data. Indeed, Feller (1971b, p. 278) shows that the class of regularly varying or "heavy tailed" dfs exhibits additivity in the tail area but not over the full distributional support. Also, the stable class fails to have a finite variance when $\alpha<2$. Finally, the normal df is a "local alternative" for the stable model which implies that stable processes with $\alpha$ only slightly smaller than 2 can hardly be distinguished from a normal df $(\alpha=2)$ on the basis of $\alpha$-estimates in very large samples. The other models we use for simulation do not suffer from these drawbacks.

In order to further mimic the time series properties of real-life financial return data, we also use models that exhibit dependence in returns and volatility. An $A R(1)$ process with nonzero first order serial correlation $\theta$ and with symmetric stable innovations is used to generate serially dependent data in order to mimic market microstructure effects in high frequency data, see e.g. Andersen and Bollerslev (1997). In order to generate persistence in volatility, we use two distinct models. First, we implement the following model to generate returns $X_{t}$ proposed by Danielsson et al. (2001):

$$
\begin{aligned}
& X_{t}=U_{t} \sqrt{\frac{v}{\chi^{2}(v)}} H_{t}, \quad P\left\{U_{t}=-1\right\}=P\left\{U_{t}=1\right\}=0.5 \\
& H_{t}=\beta Q_{t}+\theta H_{t-1}, \quad Q_{t} \sim N(0,1)
\end{aligned}
$$

where $\chi^{2}(v)$ stands for the chi-square distribution with $v$ degrees of freedom and with $(\beta, \theta)=(0.1,0.9)$. The unconditional df is Student-t with $\alpha=v$ degrees of freedom. The multiplicative factor $U_{t}$ guarantees the fair game property $E_{t-1} X_{t}=0$ while preserving the volatility clustering feature. Second, we also simulate from a $\operatorname{GARCH}(1,1)$ model with conditionally normal innovations. The sum of the GARCH volatility parameters $\theta=\beta_{0}+\beta_{1}$ is chosen such that the tail index of the corresponding unconditional df equals 4 . The GARCH model class enables one to change the volatility persistence $\theta$ ceteris paribus the tail index. ${ }^{12}$

\footnotetext{
${ }^{12}$ The unconditional distribution of a $\operatorname{GARCH}(1,1)$ process with conditionally normal standardized residuals can be shown to exhibit a heavy tail, see Mikosch and Starica (2000). They also derive a closed-form relation between the tail index and the parameters of the conditional variance equation. For $\alpha=4$ (a representative value for the tail index of financial assets in the empirical literature), the closed-form relation specializes to a quadratic equation in the GARCH parameters $\left(\beta_{0}, \beta_{1}\right)$ governing the conditional variance equation. Exact parameter values can be calculated by restricting the parameter sum $\theta=\beta_{0}+\beta_{1}$ to values below 1 .
} 
3.2. Choice of optimal number of extremes. Tail index estimators like the Hill statistic imply a bias/variance trade-off, i.e. the more data used from the distributional centre the smaller will be the variance of the estimator at the cost of an increase in bias. Goldie and Smith (1987) therefore proposed to select the number of upper order extremes $m$ used in estimating (2.2) by minimizing the Asymptotic Mean Squared Error (AMSE) of the Hill statistic. Using the second order expansion (2.1) for regularly varying tails, Danielsson and de Vries (1997) derived an expression of the AMSE of $\widehat{\alpha}$ in terms of the second order expansion parameters:

$$
\operatorname{AMSE}(\widehat{\alpha})=a^{-2 \beta / \alpha} \frac{1}{\alpha^{2}} \frac{\beta^{2} b^{2}}{(\alpha+\beta)^{2}}\left(\frac{m}{n}\right)^{\frac{2 \beta}{\alpha}}+\frac{1}{\alpha^{2} m},
$$

where the first part is the squared bias and the second part is the asymptotic variance. The above expression shows that the second order parameters $b$ and $\beta$ are responsible for the bias in the Hill statistic, i.e. if either $b$ or $\beta$ equal zero, the bias term disappears and the distributional tail (2.1) specializes to an exact Pareto.

Minimizing (3.1) w.r.t. $m$ renders the optimal number $m^{*}$ of highest order statistics

$$
m^{*}=c n^{2 \beta /(2 \beta+\alpha)}, \quad c=\left(\frac{\alpha(\alpha+\beta)^{2}}{2 \beta^{3} b^{2}} a^{2 \beta / \alpha}\right)^{\frac{\alpha}{(2 \beta+\alpha)}},
$$

which is the same expression as under condition (B) of Theorem 1.

The parameter set $(a, b, \alpha, \beta)$ - and thus the value of $m^{*}$ - can be obtained for certain distributional models by developing the tail expansion (2.1). ${ }^{13}$ However, for stochastic processes with unknown tail expansion parameters and for the real-life data in the empirical Section, the closed-form expression (3.1) for AMSE does not exist. Instead, we implement the Beirlant et al. (1999) algorithm that minimizes a sample equivalent of the AMSE. ${ }^{14}$ In order to save computation time, we did not determine the optimal nuisance parameter $m^{*}$ for each recursive,

All technical details on this closed-form expression are provided in Appendix C ("Calibration of GARCH $(1,1)$ parameters").

${ }^{13}$ This is the case for the stable, student-t, Burr and stochastic volatiliy models we considered. Details on the accompanying tail expansion derivations are included in Appendix B ("Derivation of 2nd order expansion parameters").

${ }^{14}$ Subsample bootstrap algorithms to select $m^{*}$ by means of AMSE minimization constitute an alternative route (see e.g. Danielsson et al., 2001); but these subsample strategies typically require much larger samples than the ones we use in the Monte Carlo section and the empirical application and are therefore unsuited for the present analysis. 
rolling or sequential subsample in (2.5)-(2.6)-(2.7) separately. Instead, we determined the full sample $\widehat{m}$ which automatically identifies the full sample scaling constant $c$ in (3.2) by $\widehat{c}=\widehat{m} / n^{2 / 3} \cdot{ }^{15}$ Extrapolating the optimal path for $m$ to the subsamples defined by the stability tests (and using the notation from Section 2.2), we obtain $\widehat{m}_{t}=\widehat{c} t^{2 / 3}$ for the recursive and sequential tests and $\widehat{m}_{w^{*}}=\widehat{c}\left(w^{*}\right)^{2 / 3}$ for the rolling test, respectively. For sake of simplicity we assume that $c$ does not change across subsamples and that it can be set equal to its full sample value.

3.3. Monte Carlo results. We first investigate the impact of $\alpha$-breaks on the finite sample performance of tail index and extreme downside risk measures, i.e. should we care about the detection and presence of breaks when applying EVT techniques that assume stationary tail behavior? Next, we evaluate the finite sample critical values and power of the considered stability tests for $\alpha$. We also investigate the ability of the tests to locate break dates. To this aim, we simulate from the set of models that have been introduced in the previous Section.

3.3.1. Tail index and quantile estimation in the presence of breaks. Prior to investigating the finite sample performance of the stability tests for $\alpha$, it seems natural to consider the finite sample performance of the Hill statistic and a popular quantile estimator that uses the Hill statistic as input. More specifically, we employ the semi-parametric quantile estimator introduced by de Haan et al. (1994):

$$
\widehat{q}_{p}=X_{n-m, n}\left(\frac{m}{p n}\right)^{\frac{1}{\alpha}},
$$

and where the "tail cut-off point" $X_{n-m, n}$ is the $(n-m)$-th ascending order statistic (or loosely speaking the $m$-th smallest return) from a sample of size $n$ such that $q>X_{n-m, n}$. The quantile $\widehat{q}_{p}$ is interpretable as the daily Value-at-risk (VaR) at the $p \%$ significance level. Financial extremes by definition do not strike often but if they occur they can drive financial institutions into overnight distress and jeopardize overall financial stability. Thus, looking at VaR numbers further into the tail than usual (i.e. corresponding with very low levels of $p$ ) is potentially relevant for both risk managers and regulators. As an illustration, consider the problem of allocating upper limits on open positions to foreign currency dealers by the treasurers of the forex dealing room of

\footnotetext{
${ }^{15}$ The exponent $2 / 3$ follows from imposing the restriction $\alpha=\beta$ on the tail expansion parameters. This circumvents the need of separate $\beta$-estimation. Moreover, simulations have shown that the Beirlant criterion to determine the optimal value of $m^{*}$ still performs well under this restriction even when the true values of $\beta$ and $\alpha$ differ, see e.g. Beirlant et al. (1999).
} 
an international bank. ${ }^{16}$ The trading limits depend on the probability $p$ on a single large negative currency return that can bring the bank's solvency in jeopardy. In this example, the level $p$ is interpretable as the insolvency risk the management considers "acceptable". Suppose the management chooses a critical loss level $s<0$ which stands for the maximum loss that can be incurred without running into solvency problems. A simple way to determine the maximum allowable investment $I$ is to set $I=s / \widehat{q}_{p}$ with $\widehat{q}_{p}$ the extreme quantile estimator as defined in (3.3).

Turning to the finite sample performance of the above tail index and quantile estimators, we know from Theorem 1 that both estimators ( $\widehat{\alpha}$ and $\widehat{q}_{p}$ ) are asymptotically biased under condition (B). A Monte Carlo investigation can clarify to what extent the asymptotic bias transfers into finite sample bias and estimation risk for the Hill statistic and the quantile estimator. Table 1 contains averages, standard errors and Root Mean Squared Errors (RMSE) for $\widehat{\alpha}$ and $\widehat{q}_{p}$. The Monte Carlo experiment is performed for sample sizes of 8,000 observations and for 10,000 replications. The VaR's significance level $p$ is set equal to the inverse of the sample size. ${ }^{17}$

\section{[Insert Table 1]}

Table 1 distinguishes between models that either generate dependent or independent draws (lower and upper panel, respectively). In the upper panel, we let $\alpha$ and the second order parameter $\rho=-\beta / \alpha$ vary; in the lower panel, the degree of serial correlation or volatility clustering (parameter $\theta$ ) is manipulated ceteris paribus $\alpha$ and $\beta$. The outcomes show a large heterogeneity in finite sample bias and estimation accuracy across different distributions. This reflects the differences in the second order behavior of the considered tail models. Notice that in the case of pure Pareto-type tail behavior (no second order behavior), the asymptotic bias in the Hill statistic and the corresponding quantile would be equal to zero.

Biases in the Hill statistic and the estimated quantiles necessarily exhibit opposite signs, see the quantile formula (3.3). Consistent with Theorem 1, the sign of the bias in the Hill estimator corresponds with the sign of $b$. Notice also that bias and standard error of the Hill statistic are smaller for heavier tails (lower values of $\alpha$ ). This is because lighter tails are closer to a thin tailed local alternative like the normal

\footnotetext{
${ }^{16}$ See Danielsson and de Vries (1997) for a more elaborate discussion and for other applications of extreme quantile estimation for e.g. institutional investors.

${ }^{17}$ This corresponds with extreme event magnitudes as reflected by the historical sample boundaries.
} 
distribution that does not satisfy (2.1). This decreases the accuracy of tail estimation techniques that assume regular variation as a starting point. It is also worth noticing what happens when the second order parameter $\rho$ changes for given values of $\alpha$. The Burr outcomes reveal that the bias and standard error of $\widehat{\alpha}$ decrease for higher values of $\beta$, i.e. the more the tail expansion (2.1) approximates a pure Pareto tail the smaller will be the bias and estimation risk. The lower panel of the table illustrates the impact of temporal dependence on bias and variance properties of tail index and quantile estimators. Both higher serial correlation in the $\mathrm{AR}(1)$ processes as well as a higher persistence in volatility clustering (Stochastic Volatility and GARCH model class) seem to increase bias and standard error.

Next we add $\alpha$-breaks to the simulation setup in order to see how this alters the finite sample performance of tail index and extreme quantile estimation as compared to Table 1 . The results are contained in Table 2 .

\section{[Insert Table 2]}

For sake of convenience we limit ourselves to those i.i.d. cases where the true quantile is known such that we can calculate the Root Mean Squared Error (RMSE) for the latter estimator. We either assume that tails become fatter $\left(\left(\alpha_{1}, \alpha_{2}\right)=(4,2)\right.$ or "thin-to-fat" scenario) or thinner $\left(\left(\alpha_{1}, \alpha_{2}\right)=(2,4)\right.$ or "fat-to-thin" scenario). Moreover, the within-sample location $r$ of the breaks is varied $(r=0.25,0.50,0.75)$. For sake of comparison, we also reported the previous table's tail index and quantile estimates without tail breaks. Evidently, the "true" quantile relevant for current risk management and stability assessments is the quantile based on the the post-break tail index. This e.g. implies that the outcomes for the break scenario $\left(\alpha_{1}, \alpha_{2}\right)=(4,2)$ have to be compared with the no break case of $\alpha=2$.

The main message of Table 2 is that the finite sample performance deteriorates in the presence of breaks as compared to the situation without breaks. How big the impact of breaks on finite sample performance is crucially depends on the location of the break and the direction of the $\alpha$-shifts. Nonsurprisingly, a fat-to-thin shift (thin-to-fat shift) leads to an overestimation (underestimation) of the true tail risk - as measured by the true tail quantile $q_{p}$. Moreover, the erosion in finite sample performance due to the presence of breaks seems more severe when the break occurs relatively late in the sample. Understandably, this is due to the fact that the bulk of the data used in estimating the full sample tail index and quantiles do not exhibit the currently relevant tail index. Finally, notice that the impact of $\alpha$-breaks on finite sample 
performance is more severe under the fat-to-thin regime shift. The intuition behind this result is that the Hill statistic and quantile estimation is conditional upon the $m$ largest observations such that outlier behavior from the fat tailed initial sample remains in the selection of the $m$ observations in the latter part of the sample. This is not the case under the thin-to-fat regime shift.

3.3.2. Finite sample performance of stability test. The asymptotic distribution of the considered stability tests crucially depends on the asymptotic behavior of the underlying Hill statistic described in Theorem 1. Whereas condition (A) renders one set of critical values that only applies under pure Pareto-type tail behavior, condition (B) implies that the parameter $\varphi=\operatorname{sign}(b)(2 \beta / \alpha)^{-1 / 2}$ determining the asymptotic bias of the Hill estimator also enters the asymptotic critical values. In this Section we investigate the impact of this asymptotic bias term on the critical values, power and ability to date breaks in finite samples. In theory, it is possible to calculate the biased as well as the bias-corrected asymptotic critical values with great precision provided one knows the parameter $\varphi$. As to date, however, there are no estimators for $\varphi$ that exhibit a satisfactory finite sample performance that is robust across the main types of regulary varying tail models, see e.g. Danielsson and de Vries (1997) or Gomes et al. (2002, 2003). ${ }^{18}$ Moreover, whereas the asymptotic distributions across different DGP's can only differ due to differences in $\varphi$, the finite sample critical values, power and break date estimates may also depend on the sample size $n$ and the optimal nuisance parameter $m^{*}$. Otherwise stated, we would like to know to what extent the bias of the Hill estimator influences the finite sample size and power properties of the stability tests as well as their ability to accurately identify break dates.

Tables 3 and 4 report simulated critical values for i.i.d. models and models that exhibit temporal dependence, respectively. Each table is further split in three panels for the recursive, rolling and sequential tests in (2.5)-(2.6)-(2.7). The quantiles of the test statistics are calculated as follows. For samples of size $n=500$ and 2,000 we generate 20, 000 simulations from the considered DGP's. These Monte Carlo simulations are used to obtain estimates of the 90th, 95th and 99th percentile of the stability tests' finite sample distribution.

\footnotetext{
${ }^{18}$ Quintos et al. (2001, p. 639) also propose a bias correction procedure under the restriction that $\operatorname{sign}(b)=1$. Although this sign restriction holds for the class of stable dfs, $b$ is negative for a majority of regularly varying models. The parameter $b$ can be positive or negative for real data sets which implies that the bias correction procedure of Quintos et al. (2001) increases the bias if $\operatorname{sign}(b)=-1$.
} 
[Insert Tables 3 and 4]

The heterogeneity in the finite sample critical values across different DGP's is comparable with the preceding tables on bias and estimation risk for the Hill and quantile estimators. This illustrates the fact that the DGP's under consideration deviate from pure Pareto tail behavior and also exhibit very different 2 nd order tail behavior. Critical values and their estimation risk are higher for those cases that exhibit a stronger bias in the Hill estimator. More specifically, higher values of the tail index $\alpha$ and the persistence parameter $\theta$ (either standing for serial correlation or volatility persistence) increase the critical values/estimation risk whereas higher values of the second order parameter $\rho=-\beta / \alpha$ (cf. Burr df) decrease the critical values. Thus, Tables 3 and 4 provide convincing evidence that the bias in the Hill estimator is transferred into the critical values. The critical values for $\rho=-5$ actually come close to the asymptotically unbiased critical values reported in Quintos et al. (2001). This should not surprise given the fact that the Burr tail comes close to a Pareto tail for $\rho=-5$ and that the Hill statistic is asymptotically unbiased for pure Pareto data. But Tables 3 and 4 also illustrate that using asymptotically unbiased critical values would lead to a huge overrejection of the null of parameter constancy.

Next, Tables 5 and 6 report finite sample power and estimates of the break points for the recursive, rolling and sequential stability test, respectively. We consider sudden upwards and downwards jumps in $\alpha$ of different magnitudes and at different points in time $(r=0.25,0.50,0.75)$. The power calculation is based on 20,000 replications and is size-adjusted by means of the finite sample critical values from Tables 3-4. The breakpoint estimates are also based on 20,000 replications but the reported average break point estimates are only based on "candidate"breaks $\widehat{r}$ that are statistically significant according to the $95 \%$ finite sample critical values from Tables $3-4 .{ }^{19}$

\section{[Insert Tables 5 and 6]}

The direction of change in $\alpha$ seems to be crucial for the finite sample power and ability to date breaks. The recursive and rolling test both exhibit satisfactory power if $\alpha$ decreases. However, the power of the rolling test is larger in detecting an increase in $\alpha$. The latter result can be understood by observing that (2.2) is based on the $m$ largest observations so that extremal returns occurring in the initial recursive sample will partly remain in the selection of the $m$ highest order statistics when the sample size increases. This initial extremes dominance

\footnotetext{
${ }^{19}$ For break scenarios $\left(\alpha_{1}, \alpha_{2}\right)$ we calculate the power and break estimates using the $95 \%$ finite sample critical value that correponds with $\min \left(\alpha_{1}, \alpha_{2}\right)$.
} 
when $\alpha_{1}<\alpha_{2}$ does not occur for the rolling test since the influence on $\widehat{\alpha}$ of extremal behavior that occurs in the initial sample gradually drops out when the rolling window is shifted through the total sample. The sequential test seems to do poorly, although the power differs quite a lot depending on the location of the break and the direction of the change in $\alpha$. As concerns the ability to date breaks, the recursive test clearly outperforms the other two tests for most considered DGP's provided the break scenario implies an increase in tail fatness $\left(\alpha_{1}>\alpha_{2}\right) .{ }^{20}$ However, the lack of power for one type of $\alpha$-jump can be easily resolved by performing the test both in calendar time ("forward" recursive test) as well as by inverting the sample ("backward" recursive test). Falls (rises) in $\alpha$ should then be signaled by the forward (backward) version of the recursive test. This is the strategy we will implement in the empirical application.

Sofar the general discussion on power and break date ability. Notice that there are also large differences in power results and break point detection across different DGP's. This heterogeneity can again be explained by the determinants of the bias in the Hill estimator. More specifically, higher values of the persistence parameter $\theta$ (either standing for serial correlation or volatility persistence) increase the bias in the Hill estimator and the bias in the estimated break dates but decrease the power. On the other hand, higher values of the second order parameter $\beta$ (cf. Burr df) decrease the bias in the Hill estimator and the bias in the estimated break dates but increase the power. Thus, Tables 5 and 6 provide convincing evidence that the bias in the Hill estimator is also influencing the stability tests' power and ability to date breaks. Indeed, the power for the Burr case with $\rho=-5$ lies close to $100 \%$, even in relatively small samples whereas bias and estimation risk for $\widehat{r}$ are negligibly small.

\section{EMPIRICAL RESULTS}

We want to assess whether the propensity towards financial extremes has changed over time for different asset classes in developed and emerging markets. For that purpose we use a bootstrap-based recursive version of the Quintos et al. (2001) stability test. The recursive test was shown to outperform the rolling and sequential tests in terms of finite sample power and ability to date breaks.

\footnotetext{
${ }^{20}$ The power and break date results show that satisfactory power is a necessary but not sufficient condition for accurate breakpoint detection. The rolling test for $\alpha_{1}<\alpha_{2}$ provides a nice illustration.
} 
It is well known that standard regression-based risk proxies like standard errors, CAPM- $\beta$ s or factor model loadings are not constant over time, see e.g. Phillips and Loretan (1990) and Ross et al. (2005). We would like to know whether and to what extent this instability in traditional risk measures transfers to unconditional tail risk measures like e.g. the tail index or tail quantiles evaluated far into the distributional tail. The riskiness of assets may also differ considerably across asset classes and/or regions (e.g. developed vs. emerging markets). Our empirical investigation therefore encompasses a large cross section of different asset types (stocks, bonds, commodities, foreign exchange, gold, silver and oil). Large institutional investors like pension funds in search of fresh diversification opportunities invest in a growing variety of asset classes and geographic regions and would like to know whether and to what extent these different asset tail risks change over time. Moreover, we distinguish between stock indices and foreign exchange rates of developed and emerging markets. ${ }^{21}$ Given the fact that large institutional investors increasingly invest in emerging markets, it is important to assess whether emerging market assets exhibit relatively more frequent shifts in tail behavior. This may be expected to be the case due to a less stable political and institutional environment as compared to more developed countries. ${ }^{22}$ An elaborate description of data and data sources is provided in appendix A.

The previous simulation Section illustrated that several forms of temporal dependence bias the recursive test's finite sample critical values. Upon assuming GARCH-type volatility clustering as the main source of temporal dependence, we implement a and GARCH-corrected version of the recursive test

$$
Q=\sup _{r \in R_{\tau}} \widehat{\eta}_{t}^{-1} Y_{n}^{2}(t),
$$

where $\widehat{\eta}_{t}$ is the estimate of the time varying scaling factor, see Quintos et al. (2001, p. 643). The extensive Monte Carlo simulations in the previous Section convincingly showed that the test's finite sample

\footnotetext{
${ }^{21} \mathrm{~A}$ break analysis for emerging bond markets is not performed because the latter series are not available for sufficiently long time periods.

${ }^{22} \mathrm{~A}$ logical multivariate extension would be to investigate whether and to what extent the cross-asset tail dependence shifts over time. Tail dependence can be identified either by means of copulae evaluated in the tail area or by a tail index of an auxiliary variable that summarizes the dependence structure in the tails, see e.g. Hartmann et al. (2006) for an earlier application on the multivariate dependence structure of bank stocks. Straetmans et al. (2008) investigated whether the 9/11 terrorist attacks had a significant impact on the tail dependence between U.S. sectoral indices and the market as a whole.
} 
distribution depends on the parameters of the regularly varying tail. In other words, there is no single set of critical values that applies to all return series. As a solution to this problem, we use bootstrap-based critical values at the $95 \%$ and $99 \%$ levels to determine finite sample critical values for each financial series separately. As the scaling factor in (4.1) already corrects the test for any temporal dependence, the bootstrap no longer has to take care of any temporal dependence and we can resort to a "wild" version of the bootstrap instead of a block bootstrap. We run the recursive test both in calendar time (forward test) and in reverse calendar time (backward test) in order to detect potential falls and rises in the tail index, respectively.

Results of the stability tests are contained in Table 7 for a large variety of stock indices, bond indices, exchange rates and commodities. Furthermore, we distinguish between mature and emerging stock markets and currencies. Table 7 reports tail index and extreme quantile estimates using the estimators earlier introduced in (2.2) and (3.3). ${ }^{23}$ The VaR quantiles are calculated for a marginal significance level $p$ of $0.015 \%$ such that the corresponding extreme events are expected to happen once every 6,500 days (which amounts to once every $6,500 / 260 \approx$ 25 years). ${ }^{24}$ The number of upper order extremes $m^{*}$ used in estimating the test statistic, the bootstrap-based critical values and the extreme quantiles are determined using the Beirlant et al. (1999) method. The maximum values for the forward and backward version of test (4.1) are included in the columns labelled $Q_{F}$ and $Q_{B}$, respectively. Evidently, bootstrapped critical values are identical for the forward and backward test. The null of parameter constancy is rejected if the sup-value calculated according to (4.1) exceeds the bootstrap-based critical values, e.g. $Q>C V_{B}(p)$ with $p=5 \%$ or $1 \%$. Statistically significant break dates are reported between brackets beneath the testing values $(\mathrm{dd} / \mathrm{mm} / \mathrm{yy})$.

\section{[Insert Table 7]}

First and foremost, one can see that the overall number of $\alpha$-breaks remains limited. The null hypothesis of a constant tail index $\alpha$ is only rejected at the $1 \%$ level for 6 out of 28 assets. The majority of breaks is detected in emerging currency markets. The emerging currency tail break dates confirm earlier research by Candelon et al. (2006) for a shorter sample of Asian currencies. Both the forward breaks (drops in $\alpha$ ) and backward breaks (rises in $\alpha$ ) fall within the time window of the Asian financial crisis. Moreover, the forward breaks precede the

\footnotetext{
${ }^{23}$ In case of two breaks $t_{1}<t_{2}$, the pre-break and post-break estimates are conditioned on subsamples $\left[1, t_{1}\right]$ and $\left[t_{2}, n\right]$, respectively.

${ }^{24}$ We assume that there are 260 trading days in a calendar year.
} 
backward breaks which suggests a U-shaped pattern for the tail index (and an inverted U-shape pattern for the quantile). As a result of unsustainable speculative pressures all considered Asian currency regimes were abolished during the second half of 1997. The Central Banks of Thailand, Malaysia and Indonesia all announced a managed float in the first half of July. For Thailand, the estimated forward break nearly perfectly coincides with that regime shift but the forward breaks for the other countries seem to lag behind the managed float announcement for approximately six months. ${ }^{25}$ We only find a backward break (rise in $\alpha$ ) for the Mexican Peso which can be interpreted as evidence for the success of the rescue and stabilization policies set up by the Mexican government and the IMF in the aftermath of the December 1994 devaluation of the Mexican Peso against the US $\$ .{ }^{26}$ Finally, the US stock market (stock market crash of 1987) and the UK bond market (expansive monetary policy after the 1987 stock market crash) exhibit a significant shift in $\alpha$. All other stock and bond market tails seem to exhibit stationary tail behavior.

We also report pre-break and post-break tail index and quantile values for the statistically significant breaks in $\alpha$ at the $1 \%$ level. The bulk of these extreme quantile shifts is situated in emerging currency markets. Notice that the post-break emerging tail risk drops dramatically compared to its pre-break value (except for the Thai currency). This suggests that the backward breaks (rise in the tail index) more than offset the preceding forward breaks (drops in the tail index). The stronger statistical significance (higher sup-values) for the backward breaks point in that direction indeed.

Turning to the extreme VaR estimates in Table 7's right part one first of all observes the huge cross asset differences in extreme downside risk. Exchange rate regimes in emerging markets seem completely ineffective in dampening exchange rate volatility as the VaR estimates

\footnotetext{
${ }^{25}$ This break date delay may be due to the bias in the Hill estimator that may be more severe than for other assets.

${ }^{26}$ This latter devaluation was not detected by the stability test because the test's interior region $R_{\varepsilon}=[0.15 n ; 0.85 n]$ did not contain December 1994.The same applies for potential breaks due to the 2007-2010 credit crunch. If present, they will only become identifiable when the sample gets longer and the candidate break dates fall within the interior region. However, even if breaks are inside the interior region, they are more difficult to detect when they lie close to the interior region boundaries. This is because the recursive stability test's finite sample power decreases for breaks that lie close to the interior sample boundaries, see our own simulation section results or Candelon and Lutkepohl (2001).
} 
are much larger for emerging currency regimes as compared to floating (developed) currency markets. Thus attempts towards exchange rate stabilization in emerging markets seem counterproductive over the considered sample period, see also Koedijk et al. (1992) for earlier evidence. Legal and institutional restrictions in emerging stock markets also do not help to curb emerging equity volatility although it does not seem to have such adverse effects as in forex markets. As a matter of fact, emerging and developed stock market tails seem to exhibit extreme downside risk of comparable magnitudes. Finally, mature stock markets exhibit more extreme downside risk than mature bond markets, see also de Haan et al. (1994) or Hartmann et al. (2004).

In order to better grasp the implications of non-constant downside risk for risk management, reconsider the earlier discussed EVT application of allocating upper limits on open positions in the forex dealing room of an international bank. We argued that the maximum allowable investment for forex traders in an open position equals $I=s / \widehat{q}_{p}$ for a given critical loss level $s<0$ and with $\widehat{q}_{p}$ the extreme quantile estimator as defined in (3.3). Given the break results for emerging currencies, full sample trading limits will be set too conservatively because they do not take into account the thinning of the tails due to e.g. subsequent liberalizations and abolishment of exchange rate regimes. Thus, given the unstable tail behavior of emerging currency returns, it is advisable to use shorter data windows that started more recently (ideally after a break has occurred in the tail behavior) for determining trading limits on currency positions. For example, consider a US bank trader that considers to build up a position in Mexican Peso. The superior management determines that the maximum loss the bank can suffer without running into solvency problems is 10,000,000 US\$. If the trader determines his trading limit using the full sample of Mexican Peso/US\$ quotes, the relevant full sample $0.015 \%$ VaR quantile in table 7 reads $30.28 \%$. This, in turn, induces a trading limit of $I_{\text {full }}=10,000,000 \mathrm{US} \$ / 0.3028=33,025,099 \mathrm{US} \$$. However, using the post-break $0.015 \%$ VaR quantile renders a trading limit of $I_{\text {post }}=10,000,000 \mathrm{US} \$ / 0.1109=90,171,326 \mathrm{US} \$$ which is nearly three times as high. In other words, taking into account that the extreme risk of the currency has diminished over the sample period due e.g. to regime changes also renders the maximum allowable investment less conservative. This stylized example for one currency can be easily generalized to a portfolio of currencies but the principle remains the same.

The results on the currency tails are also interesting from a policy perspective. Although many countries exhibit a fear of floating, fixing the exchange rate in one way or another by e.g. capital controls or 
other restrictions on current account and capital account convertibility seems counterproductive. The downside risk estimates, as measured by the extreme quantile $q_{p}$, are nearly always higher in the presence of regimes. The economic interpretation is that a float lets exchange rates adjust more smoothly than any other regime that involves some fixity. Monetary history indeed shows that it is extremely difficult for monetary authorities to establish and sustain perfectly credible and time-consistent forex regimes. Imperfectly credible capital controls, however, are to speculators like a red rag to a bull. The inverse relation between extreme depreciation risk and the abolishment of capital controls seems to support Friedman's old plea for flexible exchange rates, see e.g. Friedman (1953).

With an eye toward some sensitivity analysis, we also applied the recursive testing procedure on a few economically meaningful subsamples around crisis episodes like the dotcom bubble or the 1987 stock market crash. The series that do not exhibit full sample breaks are not characterized by subsample breaks either as one would expect. Evidently, subsample breaks should not necessarily be identical to the full sample breaks if the latter fall outside the selected subsample. However, when the full sample breaks do fall in the selected subsample, break results are robust and the location of the break is only marginally altered by the change in sample size.

Overall, the empirical results suggest that heavy tails and corresponding extreme quantiles are remarkably stable over long periods of time for most of the considered assets and asset classes. Tail index and extreme quantile estimation seem to be useful tools for assessing longterm tail risk, stress testing and financial stability but techniques have to be applied with care in the presence of breaks in the tail behavior. Hedging tail risk of portfolios containing large positions in emerging currencies constitutes an example.

\section{Conclusions}

This paper provided a thorough study of the finite sample behavior of some popular tests for detecting time variation in the tail index of financial returns. The tests are "endogenous" in the sense that they produce an estimate of the breakpoint location upon detection of a statistically significant break. Our Monte Carlo experiment determined critical values, size-corrected power and the ability to date breaks for a myriad of Data Generating Processes (DGP's). The tests all used the Hill estimator for the tail index as an input. Conform to the bulk of the empirical literature, the number of upper order extremes was 
selected by minimizing the sample Mean Squared error of the Hill statistic. The DGP's were chosen with an eye towards mimicking some popular empirical stylized facts of financial data. The finite sample critical values, the (size-corrected) power and the ability to date breaks differed a lot across different distributional models and sample sizes. Nonsurprisingly, our simulation experiments showed that critical values increase and the power and break date ability decreased when the bias in the Hill estimator becomes more severe. As there are no satisfactory bias reduction methods available for the finite sample critical values, we proposed a bootstrap-based procedure for the critical values of the stability test when working with real-life data. We implemented a recursive version of the stability test in the empirical application as this version outperformed its rolling and sequential counterparts in a simulation environment. Upon applying the stability test to a large set of asset classes, both in developed as well as emerging markets, we hardly detected any breaks at all, except for emerging currency tails. For those series with breaks in the tail behavior, it is advisable to base tail risk measures like VaR or expected shortfall on the post-break sample.

\section{Appendix A. Data Description and Discussion}

Data were obtained from Datastream, Inc. for different asset classes. Returns were calculated as log price differences between daily closes. Developed and emerging stock and bond indices are expressed in local currency and listed with the following abbreviations: France (FR), Germany (GE), United Kingdom (UK), United States (US), Japan (JP), Indonesia (INDO), Malaysia (MAL), Thailand (THAI), Mexico (MEX), Chile (CHIL). Emerging bond index data were excluded because of insufficient data availability. Stock indices are Datastream calculated total return indices whereas bond indices correspond to total return indices on 10-year ("benchmark") government bonds. The exchange rate data also consist of a developed and emerging currency block. All currencies are expressed against the US dollar. Initially, we downloaded nominal bilateral exchange rates against the Pound sterling (GBP) as this renders the largest cross section of currencies over the longest possible time span. Cross rates against the US dollar (USD) were calculated by applying the no triangular arbitrage condition. The industrial block covers the euro (EUR, and before January 1999 the Deutsche Mark), the Japanese Yen (JPY), the Pound sterling (GBP), the Swiss franc $(\mathrm{CHF})$ and the Canadian dollar (CND). The emerging currency block includes the Indonesian rupiah (IRD), the Malaysian ringgit (MYR), the Thai baht (THB), the Chilean peso (CLP) and the 
Mexican peso (MXN). Finally, prices of oil (Brent Crude), gold and silver are expressed in US\$ per barrel or per troy ounce, respectively.

All series end on 31 December 2009 but they exhibit different starting points due to data availability. Nonsurprisingly, emerging market data availability is limited as compared to more mature markets: developed/emerging stock market series and developed/emerging currencies start from 1 January 1973, 2 April 1990, 3 January 1972 and 3 January 1994, respectively. Bond series start in the first half of the 80s: United States, United Kingdom and Germany (1 January 1980), Japan (2 January 1984), France (2 January 1985). Finally, commodities start on 3 January 1972 (gold and silver) and 4 January 1982 (oil), respectively.

\section{ApPEndix B. DeRIVATIONS OF 2ND ORDER EXPANSION PARAMETERS}

In Theorem 1 we argued that $m=c n^{2 \beta / 2 \beta+\alpha}$ is the optimal nuisance parameter for the Hill statistic that minimzes the $\operatorname{AMSE}(\widehat{\alpha})$. The scaling constant $c$ in turn depends on the parameters $(a, b, \alpha, \beta)$ of the second order tail expansion (2.1). Thus, the parameters and the resulting $m^{*}$ are uniquely determined upon knowledge of this tail expansion. To simplify their derivation it is instructive to re-express the tail expansion (2.1) for $p=x^{-1}$ close to zero:

$$
1-G(p)=a p^{\alpha}\left(1+b p^{\beta}+o\left(p^{\beta}\right)\right),
$$

with $a>0, b \in \Re, \beta>0$ and $F(x)=G(p)$. In the Monte Carlo section we show that biases in the Hill estimator, the stability tests' critical values and the breakpoint estimates are critically determined by the level of $\mathrm{b}$ and $\beta$. The pure Pareto model $(b=0$ and/or $\beta \sim \infty)$ provides the benchmark case because it renders unbiased Hill estimates, test statistics and break point estimates.

The parameters $a, b$ and $\beta$ easily follow by either expanding the cumulative distribution $G(p)$ (c.d.f.) (if it exists in closed form) or the accompanying density around $p=0$. The Frechet and Burr dfs have c.d.f.s in closed form which implies that their respective second order taylor expansions for $\mathrm{p}$ small ( $x$ large) straightforwardly follow as

$$
\begin{aligned}
1-G_{F R E C H E T}(p) & =1-\exp \left(-p^{\alpha}\right) \\
& \simeq p^{\alpha}\left(1-\frac{1}{2} p^{\alpha}\right), \quad p \text { small } \\
& =x^{-\alpha}\left(1-\frac{1}{2} x^{-\alpha}\right), \quad x \text { large }
\end{aligned}
$$


and

$$
(a, b, \beta)_{F R E C H E T}=(1,-1 / 2, \alpha)
$$

As for the Burr distribution, the 2nd order expansion for the c.d.f. reads

$$
\begin{aligned}
1-G_{\text {BURR }}(p) & =\left(1+p^{-\beta}\right)^{-\alpha / \beta} \\
& \simeq p^{\alpha}\left(1-\frac{\alpha}{\beta} p^{\beta}\right), \quad p \text { small } \\
& =x^{-\alpha}\left(1-\frac{\alpha}{\beta} x^{-\beta}\right), \quad x \text { large }
\end{aligned}
$$

which implies

$$
(a, b)_{B U R R}=(1,-\alpha / \beta)
$$

Clearly, whereas first order and higher order behavior are related $(\beta=$ $\alpha$ ) in the Frechet case, the 2nd order parameter can be freely chosen in case of the Burr model. This implies that the Burr distribution becomes indistinguishable from a pure Pareto distribution for large $\beta$.

The other DGPs do not exhibit explicitc dfs in closed form which may somewhat complicate the derivation of the second order parameters. For the symmetric stable class neither the c.d.f. nor the density exists in closed form but we can exploit an existing tail expansion (Ibragimov and Linnik (1971, ch. 2)) in order to determine the parameters in (B.1):

$$
1-F(x)=\pi^{-1} \sum_{i=1}^{\infty}(-1)^{i} \frac{\Gamma(i \alpha)}{i ! x^{i \alpha}} \sin \left(\frac{i \alpha \pi}{2}\right), \quad x \text { large }
$$

Only considering the expanion's first two terms renders the second order approximation:

$$
1-F(x) \simeq \frac{1}{\pi} \Gamma(\alpha) \sin \left(\frac{\alpha \pi}{2}\right) x^{-\alpha}\left(1-\frac{\Gamma(2 \alpha) \sin (\alpha \pi)}{2 \Gamma(\alpha) \sin \left(\frac{\alpha \pi}{2}\right)} x^{-\alpha}\right),
$$

Thus the parameter vector that we need for determining $m^{*}$ boils down to:

$$
(a ; b ; \beta)_{S T A B L E}=\left(\frac{1}{\pi} \Gamma(\alpha) \sin \left(\frac{\alpha \pi}{2}\right) ;-\frac{\Gamma(2 \alpha) \sin (\alpha \pi)}{2 \Gamma(\alpha) \sin \left(\frac{\alpha \pi}{2}\right)} ; \alpha\right)
$$

It follows from the 2 nd order term that the restriction $\alpha=\beta$ holds for the symmetric stable class. ${ }^{27}$

\footnotetext{
${ }^{27}$ This restriction also holds for the Frechet model because it falls within the symmetric stable class of distributions.
} 
In order to determine the tail expansion parameters for the Studentt, we need to expand the tail density $g(p)$ because the c.d.f. does not exist in closed form. The asymptotic expansion for the class of regularly varying densities easily follows from (B.1):

$$
\begin{aligned}
& G^{\prime}(p)=g(p) \simeq a \alpha p^{\alpha+1}+a b(\alpha+\beta) p^{\alpha+\beta+1}, \quad p \quad \text { small } \\
& =a x^{-\alpha-1}\left(\alpha+b(\alpha+\beta) x^{-\beta-1}\right), \quad x \text { large }
\end{aligned}
$$

Upon rewriting the 2nd order Taylor expansion of the student-t density for large $x$ (small $p$ ) in the above format, we obtain:

$$
\begin{aligned}
f(x) & =\frac{\Gamma\left(\frac{\alpha+1}{2}\right)}{\Gamma\left(\frac{\alpha}{2}\right) \sqrt{\pi \alpha}}\left(1+\frac{x^{2}}{\alpha}\right)^{-\frac{\alpha+1}{2}} \\
& \simeq \frac{\Gamma\left(\frac{\alpha+1}{2}\right)}{\Gamma\left(\frac{\alpha}{2}\right) \sqrt{\pi \alpha}} \alpha^{\frac{\alpha-1}{2}} \alpha x^{-\alpha-1}\left[\alpha-\frac{\alpha^{2}(\alpha+1)}{2(\alpha+2)}(\alpha+2) x^{-3}\right], \quad x \text { large },
\end{aligned}
$$

which directly renders the parameter vector

$$
(a, b, \beta)_{S T U D E N T}=\left(\frac{\Gamma\left(\frac{\alpha+1}{2}\right)}{\Gamma\left(\frac{\alpha}{2}\right) \sqrt{\pi \alpha}} \alpha^{\frac{\alpha-1}{2}} ;-\frac{\alpha^{2}(\alpha+1)}{2(\alpha+2)}, 2\right)
$$

It follows from the second order term between brackets that the restriction $\beta=2$ holds. for the symmetric stable class.

Finally, it can be easily shown that the serially correlated stable draws (denoted by ARSTA in the tables) and the student-t draws that exhibit dependence in the second moment (SVSTU) in eq. (??) exhibit the same optimal $m^{*}$ as their I.I.D. stable and student-t counterparts. The additivity property under addition for the symmetric stable df ensures that the serially dependent stable draws exhibit the same distribution as the I.I.D. symmetric stable upon some scaling constant. ${ }^{28}$ The Student-t draws that exhibit dependence in the second moment are also identically distributed to I.I.D. student-t process upon some scaling constant. In general, a linear transform $\widetilde{X}=t X$ that changes the scaling constant leaves the tail index and the optimal value of upper order extremes invariant. This directly follows from the tail expansion

\footnotetext{
${ }^{28} \mathrm{An} \operatorname{AR}(1)$ process $X_{t}=\theta X_{t-1}+u_{t}$ with first order serial correlation $0<$ $\theta<1$ is equivalent to the $\mathrm{MA}(\infty)$ process $X_{t}=\sum_{i=0}^{\infty} \theta^{i} u_{t-i}$. If the innovations $u_{t-i}$ are i.i.d. symmetric stable, it follows from Feller (1971, ch. VIII.8) that $X_{t} \stackrel{d}{=}\left(1+\theta^{\phi}+\theta^{2 \phi}+\cdots\right) u_{t}=\left(\frac{1}{1-\theta^{\phi}}\right)^{\frac{1}{\phi}} u_{t}$.
} 
for $\widetilde{X}$ :

$$
\begin{aligned}
P\{\tilde{X}>x\} & =P\left\{X>t^{-1} x\right\} \\
& \simeq a t^{\alpha} x^{-\alpha}\left(1+b t^{\beta} x^{-\beta}\right)
\end{aligned}
$$

which implies that $\widetilde{a}=a t^{\alpha}$ and $\widetilde{b}=b t^{\beta}$. The parameters $\alpha$ and $\beta$ are left unchanged by the linear transform. Substituting $\widetilde{a}$ and $\widetilde{b}$ into $c=\left(\frac{\alpha(\alpha+\beta)^{2}}{2 \beta^{3} b^{2}} a^{2 \beta / \alpha}\right)$ leaves the value of $m^{*}=c n^{\frac{2 \beta}{2 \beta+\alpha}}$ invariant.

\section{Appendix C. Calibration of GaRCH $(1,1)$ parameters}

In order to generate the clusters of volatility feature for the conditional df, we simulated, inter alia, from a $\operatorname{GARCH}(1,1)$ process with conditionally normal disturbances (see Bollerslev (1986)). Let $X_{t}$ follow a $\operatorname{GARCH}(1,1)$ process, then

$$
\begin{aligned}
X_{t} & =\sigma_{t} Z_{t} \\
\sigma_{t}^{2} & =\beta_{0}+\beta_{1} \sigma_{t-1}^{2}+\lambda X_{t-1}^{2} \\
Z_{t} & \sim \text { i.i.d. } \mathrm{N}(0,1)
\end{aligned}
$$

It can be shown that the GARCH scheme also induces the fat tail property on the unconditional distribution of the returns, see e.g. Embrechts et al. (1997). Also, the tail index $\alpha$ is a function of the parameters of the model. Given the normality of $Z_{t}$ and provided $\beta_{1}+\lambda<1$, one can show that $\alpha$ is related to the parameters of the conditional df:

$$
E\left(\lambda Z^{2}+\beta_{1}\right)^{\alpha / 2}=1
$$

see e.g. Mikosch and Starica (1998). Empirical evidence suggests that $2<\alpha<4$ and we therefore used these boundary values in the Monte Carlo simulations. When $\alpha=2$, eq. (C.1) implies that $\lambda+\beta_{1}=1$. This still leaves an infinite number of possible parameter combinations. For sake of simplicity, we will calibrate $\left(\lambda, \beta_{1}\right)=(1 / 2,1 / 2)$ in the simulation section for the $\alpha=2$ case. As for the upper bound value $\alpha=4$, eq. (C.1) boils down to

$$
3 \lambda^{2}+2 \beta \lambda+\beta^{2}-1=0
$$

Upon substitute $\beta_{1}=c-\lambda(c<1)$ into (C.2), one obtains a quadratic equation in $\lambda$ :

$$
2 \lambda^{2}=1-c^{2}
$$

It follows that for a given value of $c$, the clusters of volatility parameters in the $\operatorname{GARCH}(1,1)$ model are uniquely identified, i.e., $\left(\lambda, \beta_{1}\right)=$ $\left(\sqrt{\frac{1-c^{2}}{2}}, c-\sqrt{\frac{1-c^{2}}{2}}\right)$. In empirical studies one often encounters $\beta_{1}+\lambda$ 
close to 1 . We therefore set $c$ equal to $0.75,0.85$, or 0.95 in the Monte Carlo section to investigate the impact of different degrees of volatility persistence on the test statistics. The intercept $\beta_{0}$ is set to $10^{-6}$ which is in line with previous simulation studies, see e.g. Danielsson and de Vries (1999).

\section{REFERENCES}

[1] Andersen TG, Bollerslev T. 1997. Intraday periodicity and volatility persistence in financial markets. Journal of Empirical Finance 4(2-3): 115-158.

[2] Andrews D. 1993. Tests for parameter stability and structural change with unknown change point. Econometrica 59: 817-858.

[3] Beirlant J, Dierckx G, Goegebeur Y, Matthys G. 1999. Tail Index Estimation and an Exponential Regression Model. Extremes 2(2): 177-200.

[4] Candelon B, Lütkepohl H. 2001. On the reliability of Chow type tests for parameter constancy in multivariate dynamic models. Economics Letters $\mathbf{7 3}(2)$ : $155-160$.

[5] Candelon B, Straetmans S. 2006. Testing for multiple regimes in the tail behavior of emerging currency returns. Journal of International Money and Finance 25(7): 1187-1205.

[6] Danielsson J, de Haan L, Peng L, de Vries CG. 2001. Using a bootstrap method to choose the sample fraction in tail index estimation. Journal of Multivariate Analysis 76(2): 226-248.

[7] Danielsson J, de Vries CG. 1997. Tail index and quantile estimation with very high frequency data. Journal of Empirical Finance 4(2-3): 241-257.

[8] de Haan L, Jansen DW, Koedijk KG, de Vries CG. 1994. Safety first portfolio selection, extreme value theory and long run asset risks. in Proceedings from a Conference on Extreme Value Theory and Applications, Galambos J (ed). Kluwer Press; 471-487.

[9] Dumouchel WH. 1983. Estimating the stable index $\alpha$ in Order to Measure Tail Thickness: A critique. Annals of Statistics 11(4): 1019-1031.

[10] Embrechts P, Klüppelberg C, Mikosch T. 1997. Modelling Extremal Events. Springer: Berlin.

[11] Feller W. 1971a. An Introduction to Probability Theory and its Applications (Volume I). Wiley, 3rd edition.

[12] Feller W. 1971b. An Introduction to Probability Theory and its Applications (Volume II). Wiley, 2nd edition.

[13] Friedman M. 'The Case for Flexible Exchange Rates,' Essays in Positive Economics, University of Chicago Press, 1053, 157-203.

[14] Galbraith JW, Zernov S. 2004. Circuit breakers and the tail index of equity returns. Journal of Financial Econometrics 2:109-129.

[15] Goldie CM, Smith R. 1987. Slow variation with remainder: Theory and applications. Quarterly Journal of Mathematics 38: 45-71.

[16] Gomes MI, Martins MJ. 2002. Asymptotically unbiased estimators of the tail index based on external estimation of the second order parameter. Extremes 5(1): 5-31. 
[17] Gomes MI, Haan L de, Peng L. 2003. Semi-parametric estimation of the second order parameter in statistics of extremes. Extremes 5(4): 387-414.

[18] Haan L de, Jansen DW, Koedijk KG, de Vries CG. 1994. Safety first portfolio selection, extreme value theory and long run asset risks. in Proceedings from a Conference on Extreme Value Theory and Applications, Galambos J (ed). Kluwer Press; 471-487.

[19] Haan L de, Stadtmüller U. 1996. Generalized Regular Variation of Second Order. Journal of the Australian Mathematical Society (Series A) 61: 381395.

[20] Haeusler E, Teugels J. 1985. On asymptotic normality of Hill's estimator for the exponent of regular variation. Annals of Statistics 13: 743-756.

[21] Hall P. 1982. On some simple estimates of an exponent of regular variation. Journal of the Royal Statistical Society (Series B) 42: 37-42.

[22] Hartmann P, Straetmans S, de Vries CG. 2003. A global perspective on extreme currency linkages. In Asset Price Bubbles: Implications for Monetary, Regulatory and International Policies, Hunter WC, Kaufman GG, Pomerleano M. (eds.) MIT Press: Cambridge (MA): 361-383.

[23] Hartmann P, Straetmans S, Vries CG de. 2004. Asset market linkages in crisis periods. Review of Economics and Statistics 86(1): 313-326.

[24] Hartmann P, Straetmans S, Vries CG de. 2006. Banking System Stability: a Cross Atlantic Perspective. In the Risk of Financial Institutions, Carey M, Stulz RM. (eds.) The University of Chicago Press (Chicago and London): 133193.

[25] Hill BM. 1975. A simple general approach to inference about the tail of a distribution. The Annals of Statistics 3(5): 1163-1173.

[26] Hols MCAB, de Vries CG. 1991. The limiting distribution of extremal exchange rate returns. Journal of Applied Econometrics 6(3): 287-302.

[27] Ibragimov IA, Linnik YV. 1971. Independent and stationary sequences of random variables. Wolters-Noordhof: Groningen.

[28] Jansen DW, Koedijk KG, Vries CG de. 2000. Portfolio selection with limited downside risk. Journal of Empirical Finance 7(3-4): 247-269.

[29] Jansen DW, Vries CG de. 1991. On the frequency of large stock returns: putting booms and busts into perspective. Review of Economics and Statistics 73(1): 19-24.

[30] Jondeau E, Rockinger M. 2003. Testing for differences in the tails of stockmarket returns. Journal of Empirical Finance 10(5): 559-581.

[31] Koedijk KG, Schafgans MMA, Vries CG de. 1990. The tail index of exchange rate returns. Journal of International Economics 29(1-2): 93-108.

[32] Koedijk KG, Stork PA, Vries CG de. 1992. Foreign exchange rate regime differences viewed from the tails. Journal of International Money and Finance 11(5): $462-473$.

[33] Leadbetter MR, Lindgren G, Rootzén H. 1983. Extremes and related properties of random sequences and processes. Springer-Verlag: Berlin.

[34] Longin FM. 1996. The asymptotic distribution of extreme stock market returns. Journal of Business 69(3): 383-408.

[35] Lux T. 1996. The stable Paretian Hypothesis and the frequency of large returns: an examination of major German stocks. Applied Financial Economics 6(6), 463-475. 
[36] Mandelbrot B. 1963. The variation of certain speculative prices. Journal of Business 36(4): 394-419.

[37] Mikosch T, Starica C. 2000. Limit theory for the sample autocorrelations and extremes of a GARCH(1,1) process. Annals of Statistics 28(5): 1427-1451.

[38] Pagan AR, Schwert GW. 1990. Testing for Covariance Stationarity in Stock Market Data. Economics Letters 33: 165-170.

[39] Phillips PCB, Loretan M. 1990. Testing Covariance Stationarity under Moment Condition Failure with an Application to Common Stock Returns. Cowles Foundation Discussion Paper nr. 947.

[40] Quandt R. 1960. Test of the Hypothesis that a Linear Regression obeys Two Separate Regimes. Journal of the American Statistical Association 55(290): 324-330.

[41] Quintos C, Fan Z, Phillips P. 2001. Structural Change Tests in Tail Behaviour and the Asian Crisis. Review of Economic Studies 68: 633-663.

[42] Ross SA, Westerfield RW, Jaffe J. 2005. Corporate Finance. McGraw-Hill (7th International Edition).

[43] Samorodnitsky G, Taqqu M. 1994. Stable Non-Gaussian Random Processes. Chapman and Hall: New York.

[44] Straetmans STM, Verschoor WFC, Wolff CCP. 2008. Extreme U.S. Stock Market Fluctuations in the Wake of 9/11. Journal of Applied Econometrics 23(1): $17-42$.

[45] Wagner N. 2005. Autoregressive conditional tail behavior and results on government bond yield spreads. International Review of Financial Analysis 14: $247-261$

[46] Werner T, Upper C. 2002. Time variation in the tail behaviour of Bund futures returns, Working Paper, European Central Bank. 
TABLE 1. Tail index and quantile estimation in the absence of breaks

\begin{tabular}{|c|c|c|c|c|c|c|c|}
\hline \multirow[t]{2}{*}{ DGP } & \multicolumn{3}{|c|}{ Tail index est. } & \multicolumn{3}{|c|}{ Quantile est. } & \multirow[t]{2}{*}{ True $\mathrm{q}$} \\
\hline & $\widehat{\alpha}$ & s.e. $(\widehat{\alpha})$ & $\operatorname{RMSE}(\widehat{\alpha})$ & $\widehat{q}$ & s.e. $(\widehat{q})$ & $\operatorname{RMSE}(\widehat{q})$ & \\
\hline \multicolumn{8}{|c|}{ Panel A: i.i.d. models } \\
\hline \multicolumn{8}{|l|}{ Stable } \\
\hline$\alpha=1.2$ & 1.23 & 0.06 & 0.03 & 593.57 & 155.43 & & \\
\hline$\alpha=1.5$ & 1.60 & 0.15 & & 128.23 & 38.92 & & \\
\hline \multicolumn{8}{|l|}{$\operatorname{Student}(\alpha)$} \\
\hline 2 & 1.91 & 0.12 & 0.15 & 70.84 & 13.30 & 15.32 & 63.23 \\
\hline 4 & 3.60 & 0.41 & 0.60 & 13.16 & 1.88 & 2.06 & 12.31 \\
\hline \multicolumn{8}{|l|}{$\operatorname{Burr}(\alpha,-\rho)$} \\
\hline$(2,-1)$ & 1.94 & 0.08 & 0.10 & 97.22 & 14.05 & 16.07 & 89.44 \\
\hline$(2,-5)$ & 1.99 & 0.03 & 0.03 & 91.28 & 6.04 & 6.31 & 89.44 \\
\hline$(4,-1)$ & 3.88 & 0.17 & 0.21 & 9.81 & 0.70 & 0.78 & 9.46 \\
\hline$(4,-5)$ & 3.98 & 0.06 & 0.06 & 9.54 & 0.31 & 0.32 & 9.46 \\
\hline \multicolumn{8}{|c|}{ Panel B: dependence in the first or second moment } \\
\hline \multicolumn{8}{|c|}{$e_{1}$} \\
\hline$(1.5,0.2)$ & 1.61 & 0.16 & 0.20 & 135.11 & 49.57 & & \\
\hline$(1.5,0.4)$ & 1.62 & 0.19 & 0.23 & 146.75 & 70.23 & & \\
\hline \multicolumn{8}{|l|}{$\operatorname{SVSTU}(\alpha, \theta)$} \\
\hline$(4,0.85)$ & 3.56 & 0.41 & 0.60 & 13.19 & 1.89 & 2.09 & 12.31 \\
\hline$(4,0.95)$ & 3.57 & 0.42 & 0.60 & 13.16 & 1.90 & 2.08 & 12.31 \\
\hline \multicolumn{8}{|l|}{$\operatorname{GARCH}(\alpha, \theta)$} \\
\hline$(4,0.85)$ & 3.57 & 0.41 & 0.59 & 7.28 & 1.46 & & \\
\hline$(4,0.95)$ & 3.58 & 0.50 & 0.65 & 7.54 & 4.08 & & \\
\hline
\end{tabular}


TABLE 2. Tail index and quantile estimation under the presence of breaks

\begin{tabular}{|c|c|c|c|c|c|c|c|c|}
\hline \multirow[t]{2}{*}{ DGP } & \multirow{2}{*}{$\begin{array}{c}\text { Break } \\
\left(\alpha_{1}, \alpha_{2}\right)\end{array}$} & \multicolumn{3}{|c|}{ Tail Index est. } & \multicolumn{3}{|c|}{ Quantile est. } & \multirow[t]{2}{*}{ True $\mathrm{q}$} \\
\hline & & $\widehat{\alpha}$ & s.e. $(\widehat{\alpha})$ & $\operatorname{RMSE}(\widehat{\alpha})$ & $\widehat{q}$ & s.e. $(\widehat{q})$ & $\operatorname{RMSE}(\widehat{q})$ & \\
\hline \multicolumn{9}{|c|}{ Panel A: Student } \\
\hline - no break & $(2,2)$ & 1.91 & 0.12 & 0.15 & 70.89 & 13.27 & 15.32 & 63.23 \\
\hline$-\mathrm{r}=0.25$ & $(4,2)$ & 1.98 & 0.12 & 0.13 & 57.32 & 10.24 & 11.82 & 63.23 \\
\hline$-\mathrm{r}=0.50$ & $(4,2)$ & 2.14 & 0.14 & 0.19 & 42.37 & 7.32 & 22.11 & 63.23 \\
\hline$-\mathrm{r}=0.75$ & $(4,2)$ & 2.44 & 0.16 & 0.47 & 27.49 & 4.17 & 35.98 & 63.23 \\
\hline - no break & $(4,4)$ & 3.57 & 0.42 & 0.60 & 13.14 & 1.89 & 2.07 & 12.31 \\
\hline$-\mathrm{r}=0.25$ & $(2,4)$ & 2.45 & 0.16 & 1.56 & 27.46 & 4.16 & 15.71 & 12.31 \\
\hline$-\mathrm{r}=0.50$ & $(2,4)$ & 2.13 & 0.14 & 1.87 & 42.41 & 7.37 & 30.99 & 12.31 \\
\hline$-\mathrm{r}=0.75$ & $(2,4)$ & 1.98 & 0.13 & 2.02 & 57.56 & 10.41 & 46.43 & 12.31 \\
\hline \multicolumn{9}{|c|}{ Panel B: $\operatorname{Burr}(\rho=-1)$} \\
\hline - no break & $(2,2)$ & 1.94 & 0.08 & 0.10 & 96.76 & 13.81 & 15.63 & 89.44 \\
\hline$-r=0.25$ & $(4,2)$ & 1.95 & 0.09 & 0.09 & 82.82 & 11.89 & 13.61 & 89.35 \\
\hline$-r=0.50$ & $(4,2)$ & 2.02 & 0.09 & 0.09 & 62.79 & 8.92 & 28.09 & 89.35 \\
\hline$-r=0.75$ & $(4,2)$ & 2.32 & 0.11 & 0.34 & 34.58 & 4.50 & 55.04 & 89.35 \\
\hline - no break & $(4,4)$ & 3.88 & 0.17 & 0.21 & 9.83 & 0.69 & 0.78 & 9.46 \\
\hline$-r=0.25$ & $(2,4)$ & 2.32 & 0.11 & 1.68 & 34.54 & 4.49 & 25.49 & 9.46 \\
\hline$-r=0.50$ & $(2,4)$ & 2.02 & 0.09 & 1.98 & 62.71 & 8.83 & 53.98 & 9.46 \\
\hline$-\mathrm{r}=0.75$ & $(2,4)$ & 1.95 & 0.09 & 2.05 & 82.66 & 11.85 & 74.15 & 9.46 \\
\hline \multicolumn{9}{|c|}{ Panel C: $\operatorname{Burr}(\rho=-5)$} \\
\hline - no break & $(2,2)$ & 1.990 & 0.03 & 0.03 & 91.24 & 5.99 & 6.25 & 89.44 \\
\hline$-r=0.25$ & $(4,2)$ & 2.155 & 0.03 & 0.16 & 62.57 & 3.92 & 27.15 & 89.44 \\
\hline$-r=0.50$ & $(4,2)$ & 2.451 & 0.04 & 0.45 & 37.45 & 2.14 & 52.04 & 89.44 \\
\hline$-r=0.75$ & $(4,2)$ & 2.977 & 0.05 & 0.98 & 19.84 & 0.95 & 69.61 & 89.44 \\
\hline - no break & $(4,4)$ & 3.980 & 0.06 & 0.07 & 9.55 & 0.31 & 0.33 & 9.46 \\
\hline$-\mathrm{r}=0.25$ & $(2,4)$ & 2.978 & 0.05 & 1.02 & 19.81 & 0.94 & 10.40 & 9.46 \\
\hline$-r=0.50$ & $(2,4)$ & 2.451 & 0.04 & 1.54 & 37.41 & 2.12 & 28.03 & 9.46 \\
\hline$-\mathrm{r}=0.75$ & $(2,4)$ & 2.156 & 0.03 & 1.84 & 62.46 & 3.90 & 53.14 & 9.46 \\
\hline
\end{tabular}

Note: the Monte Carlo experiment is performed for samples of size $n=8,000$. The number of replications equals 10,000. The location of the breaks is represented by r. The table reports averages, standard errors (s.e.) and Root Mean squared Errors (RMSE) for the tail index and the tail quantile estimates. 
TABLE 3. Small sample critical values for recursive, rolling and sequential tests: i.i.d. models

\begin{tabular}{|c|c|c|c|c|c|c|}
\hline \multirow[t]{2}{*}{ DGP } & \multicolumn{3}{|c|}{$\mathrm{n}=500$} & \multicolumn{3}{|c|}{$\mathrm{n}=2,000$} \\
\hline & 0.90 & 0.95 & 0.99 & 0.90 & 0.95 & 0.99 \\
\hline & \multicolumn{6}{|c|}{ Panel A: Recursive test } \\
\hline \multicolumn{7}{|l|}{$\operatorname{Stable}(\alpha)$} \\
\hline 1.2 & $1.97(0.04)$ & $2.78(0.08)$ & $5.22(0.20)$ & $2.00(0.02)$ & $2.67(0.03)$ & $4.64(0.19)$ \\
\hline 1.5 & $5.12(0.13)$ & $8.39(0.19)$ & $20.37(1.27)$ & $3.41(0.10)$ & $4.97(0.20)$ & $9.61(0.63)$ \\
\hline \multicolumn{7}{|l|}{ Student $(\alpha)$} \\
\hline 2 & $1.99(0.05)$ & $2.85(0.06)$ & $5.80(0.26)$ & $1.84(0.02)$ & $2.43(0.04)$ & $4.24(0.15)$ \\
\hline 4 & $2.42(0.08)$ & $3.87(0.21)$ & $9.20(0.81)$ & $2.18(0.04)$ & $3.17(0.08)$ & $6.33(0.34)$ \\
\hline \multicolumn{7}{|l|}{$\operatorname{Burr}(\alpha, \rho)$} \\
\hline$(2,-1)$ & $1.81(0.03)$ & $2.43(0.03)$ & $4.35(0.19)$ & $1.80(0.02)$ & $2.29(0.03)$ & $3.69(0.12)$ \\
\hline$(2,-5)$ & $1.54(0.03)$ & $1.95(0.04)$ & $3.07(0.09)$ & $1.56(0.01)$ & $1.93(0.01)$ & $2.84(0.07)$ \\
\hline & \multicolumn{6}{|c|}{ Panel B: Rolling test $(\gamma=0.2)$} \\
\hline \multicolumn{7}{|l|}{$\operatorname{Stable}(\alpha)$} \\
\hline 1.2 & $2.40(0.07)$ & $3.33(0.08)$ & $5.98(0.22)$ & $2.33(0.05)$ & $3.00(0.10)$ & $4.82(0.18)$ \\
\hline 1.5 & $14.20(0.54)$ & $22.44(1.18)$ & $54.82(4.69)$ & $6.12(0.13)$ & $8.33(0.26)$ & $14.84(0.79)$ \\
\hline \multicolumn{7}{|l|}{$\operatorname{Student}(\alpha)$} \\
\hline 2 & $2.87(0.04)$ & $4.10(0.11)$ & $7.97(0.44)$ & $2.15(0.05)$ & $2.87(0.08)$ & $4.84(0.22)$ \\
\hline 4 & $4.81(0.19)$ & $7.46(0.31)$ & $17.66(1.10)$ & $3.06(0.07)$ & $4.38(0.15)$ & $8.40(0.34)$ \\
\hline \multicolumn{7}{|l|}{$\operatorname{Burr}(\alpha, \rho)$} \\
\hline$(2,-1)$ & $1.95(0.01)$ & $2.64(0.03)$ & $4.64(0.16)$ & $1.73(0.02)$ & $2.22(0.04)$ & $3.51(0.06)$ \\
\hline$(2,-5)$ & $1.66(0.01)$ & $2.10(0.03)$ & $3.25(0.09)$ & $1.53(0.01)$ & $1.82(0.02)$ & $2.55(0.05)$ \\
\hline \multicolumn{7}{|c|}{ Panel C: sequential test } \\
\hline \multicolumn{7}{|l|}{$\operatorname{Stable}(\alpha)$} \\
\hline 1.2 & $21.67(0.53)$ & $31.73(0.86)$ & $59.01(1.89)$ & $16.21(0.45)$ & $22.54(0.96)$ & $40.38(1.98)$ \\
\hline 1.5 & $24.33(0.73)$ & $39.03(1.53)$ & $87.89(3.10)$ & $16.51(0.40)$ & $24.13(1.12)$ & $48.81(2.29)$ \\
\hline \multicolumn{7}{|l|}{$\operatorname{Student}(\alpha)$} \\
\hline 2 & $21.49(0.34)$ & $31.54(1.04)$ & $60.26(3.62)$ & $17.86(0.43)$ & $25.18(0.80)$ & $45.70(1.22)$ \\
\hline 4 & $25.05(0.47)$ & $38.41(0.77)$ & $77.96(3.55)$ & $19.04(0.67)$ & $28.16(1.06)$ & $53.39(2.55)$ \\
\hline \multicolumn{7}{|l|}{$\operatorname{Burr}(\alpha, \rho)$} \\
\hline$(2,-1)$ & $19.03(0.33)$ & $27.13(0.48)$ & $49.78(1.60)$ & $16.80(0.31)$ & $23.09(0.61)$ & $39.84(1.17)$ \\
\hline$(2,-5)$ & $20.14(0.24)$ & $27.72(0.59)$ & $49.08(1.21)$ & $19.75(0.21)$ & $26.37(0.42)$ & $43.87(0.88)$ \\
\hline
\end{tabular}

Note: critical values are reported for varying sample sizes (n), and different levels of statistical significance. Critical values are based on 20,000 Monte Carlo replications. Corresponding standard errors for the critical values are reported between brackets (s.e.). The parameters $\alpha$ and $\rho=-\beta / \alpha$ refer to the tail index and the second order parameter, respectively. 
TABLE 4. Small sample critical values for recursive, rolling and sequential tests: dependent models

\begin{tabular}{|c|c|c|c|c|c|c|}
\hline \multirow[t]{2}{*}{ DGP } & \multicolumn{3}{|c|}{$n=500$} & \multicolumn{3}{|c|}{$n=2,000$} \\
\hline & 0.90 & 0.95 & 0.99 & 0.90 & 0.95 & 0.99 \\
\hline & \multicolumn{6}{|c|}{ Panel A: Recursive test } \\
\hline $\operatorname{ARSTA}(\alpha, \theta)$ & & & & & & \\
\hline$(1.2,0.2)$ & $2.65(0.07)$ & $4.04(0.13)$ & $8.73(0.71)$ & $2.65(0.05)$ & $3.74(0.07)$ & $6.97(0.27)$ \\
\hline$(1.2,0.4)$ & $4.01(0.10)$ & $6.43(0.23)$ & $15.05(1.02)$ & $4.01(0.08)$ & $5.97(0.13)$ & $11.79(0.64)$ \\
\hline \multicolumn{7}{|l|}{$\operatorname{SVSTU}(\alpha, \theta)$} \\
\hline$(2,0.85)$ & $2.25(0.04)$ & $3.27(0.08)$ & $6.67(0.36)$ & $1.92(0.04)$ & $2.56(0.05)$ & $4.59(0.18)$ \\
\hline$(2,0.95)$ & $2.56(0.06)$ & $3.82(0.12)$ & $7.96(0.47)$ & $2.05(0.05)$ & $2.78(0.06)$ & $4.95(0.23)$ \\
\hline \multicolumn{7}{|l|}{$\operatorname{GARCH}(\alpha, \theta)$} \\
\hline$(4,0.85)$ & $3.41(0.15)$ & $6.08(0.31)$ & $20.46(1.68)$ & $2.63(0.03)$ & $3.42(0.06)$ & $7.25(0.71)$ \\
\hline$(4,0.95)$ & $4.14(0.14)$ & $7.67(0.30)$ & $26.20(2.50)$ & $3.33(0.07)$ & $5.05(0.21)$ & $15.30(1.63)$ \\
\hline & \multicolumn{6}{|c|}{ Panel B: Rolling test } \\
\hline \multicolumn{7}{|l|}{$\operatorname{ARSTA}(\alpha, \theta)$} \\
\hline$(1.2,0.2)$ & $3.16(0.05)$ & $4.40(0.11)$ & $8.26(0.49)$ & $3.12(0.08)$ & $4.07(0.08)$ & $6.54(0.24)$ \\
\hline$(1.2,0.4)$ & $4.64(0.07)$ & $6.63(0.15)$ & $12.70(0.46)$ & $4.75(0.08)$ & $6.26(0.09)$ & $10.53(0.34)$ \\
\hline \multicolumn{7}{|l|}{$\operatorname{SVSTU}(\alpha, \theta)$} \\
\hline$(2,0.85)$ & $3.24(0.10)$ & $4.54(0.20)$ & $8.97(0.42)$ & $2.27(0.04)$ & $3.04(0.05)$ & $5.05(0.24)$ \\
\hline$(2,0.95)$ & $3.73(0.08)$ & $5.22(0.13)$ & $9.87(0.43)$ & $2.46(0.05)$ & $3.25(0.09)$ & $5.48(0.16)$ \\
\hline \multicolumn{7}{|l|}{$\operatorname{GARCH}(\alpha, \theta)$} \\
\hline$(4,0.85)$ & $4.86(0.08)$ & $8.31(0.26)$ & $25.80(1.84)$ & $2.05(0.03)$ & $2.71(0.06)$ & $5.66(0.26)$ \\
\hline \multirow[t]{2}{*}{$(4,0.95)$} & $5.75(0.10)$ & $9.81(0.34)$ & $29.06(2.28)$ & $2.89(0.08)$ & $4.31(0.14)$ & $10.31(0.61)$ \\
\hline & \multicolumn{6}{|c|}{ Panel C: sequential test } \\
\hline $\operatorname{ARSTA}(\alpha, \theta)$ & & & & & & \\
\hline$(1.2,0.2)$ & $26.86(0.78)$ & $40.68(1.30)$ & $85.68(2.97)$ & $21.41(0.42)$ & $31.06(0.99)$ & $60.6(3.97)$ \\
\hline$(1.2,0.4)$ & $35.98(0.64)$ & $56.60(2.13)$ & $133.09(5.63)$ & $30.33(0.45)$ & $46.62(0.77)$ & $100.18(4.54)$ \\
\hline $\operatorname{SVSTU}(\alpha, \theta)$ & & & & & & \\
\hline $\mathrm{SV}(2,0.85)$ & $21.24(0.68)$ & $31.60(0.95)$ & $61.84(2.80)$ & $17.84(0.33)$ & $25.34(0.63)$ & $45.50(2.34)$ \\
\hline $\mathrm{SV}(2,0.95)$ & $21.25(0.59)$ & $31.54(0.95)$ & $60.88(2.34)$ & $17.72(0.33)$ & $25.15(0.58)$ & $45.62(2.17)$ \\
\hline $\operatorname{GARCH}(\alpha, \theta)$ & & & & & & \\
\hline$(4,0.85)$ & $38.55(1.11)$ & $59.42(2.31)$ & $123.01(3.55)$ & $38.11(0.88)$ & $57.20(1.01)$ & $117.21(4.69)$ \\
\hline$(4,0.95)$ & $36.76(0.97)$ & $57.25(1.92)$ & $119.74(6.98)$ & $214.97(0.90)$ & $264.68(1.05)$ & $396.78(6.50)$ \\
\hline
\end{tabular}

Note: critical values are reported for varying sample sizes (n), and different levels of significance. Critical values are based on 20,000 Monte Carlo replications. Corresponding standard errors for the critical values are reported between brackets (s.e.). The first order serial correlation of an autoregressive process with stable innovations (ARSTA), the volatility persistence parameter in GARCH $(1,1)$ models and in stochastic volatility models with student-t innovations (SVSTU) is always denoted by $\theta$. 
TABLE 5. Size-corrected finite sample power for recursive, rolling and sequential tests

\begin{tabular}{|c|c|c|c|c|c|c|}
\hline \multirow[t]{2}{*}{$\operatorname{DGP}\left(\alpha_{1} ; \alpha_{2}\right)$} & \multicolumn{3}{|c|}{$n=500$} & \multicolumn{3}{|c|}{$n=2,000$} \\
\hline & $r=0.25$ & $r=0.5$ & $r=0.75$ & $r=0.25$ & $r=0.50$ & $r=0.75$ \\
\hline \multicolumn{7}{|l|}{ Stable $(1.5,1.2)$} \\
\hline - rec & 22 & 32 & 25 & 53 & 71 & 55 \\
\hline - rol & 7 & 8 & 5 & 32 & 38 & 22 \\
\hline - seq & 14 & 28 & 42 & 15 & 45 & 69 \\
\hline \multicolumn{7}{|l|}{ Stable $(1.2,1.5)$} \\
\hline - rec & 1.18 & 1.36 & 1.5 & 1.96 & 2.66 & 1.1 \\
\hline - rol & 5 & 9 & 7 & 22 & 37 & 30 \\
\hline - seq & 6 & 3 & 1 & 10 & 4 & 2 \\
\hline \multicolumn{7}{|l|}{ Student $(4,2)$} \\
\hline - rec & 21 & 32 & 24 & 49 & 73 & 62 \\
\hline - rol & 6 & 6 & 4 & 27 & 32 & 15 \\
\hline - seq & 10 & 21 & 35 & 12 & 41 & 71 \\
\hline \multicolumn{7}{|l|}{ Student $(2,4)$} \\
\hline - rec & 0.5 & 0.7 & 2 & 2.54 & 0.94 & 1.18 \\
\hline - rol & 4 & 6 & 5 & 15 & 31 & 27 \\
\hline - seq & 3 & 1 & 0.4 & 0.6 & 0.1 & 0.6 \\
\hline \multicolumn{7}{|l|}{$\operatorname{Burr}(4,2)$} \\
\hline \multicolumn{7}{|l|}{$\rho=-1$} \\
\hline - rec & 31 & 37 & 26 & 52 & 66 & 53 \\
\hline - rol & 19 & 22 & 15 & 35 & 45 & 30 \\
\hline - seq & 17 & 41 & 57 & 14 & 45 & 68 \\
\hline \multicolumn{7}{|l|}{ Burr $(2,4)$} \\
\hline \multicolumn{7}{|l|}{$\rho=-1$} \\
\hline - rec & 1.16 & 0.5 & 1.3 & 0.3 & 0.22 & 1.76 \\
\hline - rol & 15 & 21 & 19 & 32 & 46 & 36 \\
\hline - seq & 1 & 0.2 & 0.08 & 0.28 & 0.02 & 0.1 \\
\hline \multicolumn{7}{|l|}{$\operatorname{SVSTU}(4,2)$} \\
\hline \multicolumn{7}{|l|}{$\theta=0.95$} \\
\hline - rec & 20 & 31 & 23 & 49 & 71 & 59 \\
\hline - rol & 12 & 22 & 16 & 43 & 66 & 55 \\
\hline - seq & 10 & 22 & 36 & 12 & 43 & 71 \\
\hline \multicolumn{7}{|l|}{$\operatorname{SVSTU}(2,4)$} \\
\hline - rec & 0.56 & 0.70 & 1.80 & 3.16 & 1.84 & 1.16 \\
\hline - rol & 0.2 & 0.26 & 0.66 & 1.28 & 0.74 & 0.8 \\
\hline - seq & 1.82 & 1.1 & 1.98 & 0.34 & 0.20 & 0.64 \\
\hline \multicolumn{7}{|l|}{$\operatorname{ARCH}(4,2)$} \\
\hline - rec & 6.2 & 16.74 & 22.18 & 17.42 & 31.54 & 22.56 \\
\hline - rol & 2.7 & 3.62 & 4.16 & 7.94 & 15.86 & 22.16 \\
\hline - seq & 6.72 & 10 & 14.88 & 9.14 & 20.24 & 40.6 \\
\hline \multicolumn{7}{|l|}{$\operatorname{ARCH}(2,4)$} \\
\hline - rec & 0.34 & 2.86 & 2.7 & 0.06 & 0.36 & 0.94 \\
\hline - rol & 2.82 & 1.8 & 1.2 & 5.4 & 3.44 & 2.06 \\
\hline - seq & 1.30 & 0.90 & 1.74 & 0.32 & 0.08 & 0.54 \\
\hline
\end{tabular}

Note: the power is reported for different sample sizes $(n=500,2000)$, different locations of the (true) breakpoints $(\mathrm{r}=0.25,0.50,0.75)$ and different jump scenarios $\left(\alpha_{1}, \alpha_{2}\right)$ for the tail index. The power is size-corrected using finite sample critical values and is calculated as the rejection frequency under the null hypothesis of parameter constancy using 20,000 Monte Carlo replications. The parameters $\alpha$ and $\rho=-\beta / \alpha$ refer to the tail index and the second order parameter, respectively. The volatility persistence parameter in the stochastic volatility models with student- $t$ innovations (SVSTU) is denoted by $\theta$. 
TABLE 6. Breakpoint estimates for recursive, rolling and sequential tests

\begin{tabular}{|c|c|c|c|c|c|c|}
\hline \multirow[t]{2}{*}{$\operatorname{DGP}\left(\alpha_{1} ; \alpha_{2}\right)$} & \multicolumn{3}{|c|}{$n=500$} & \multicolumn{3}{|c|}{$n=2,000$} \\
\hline & $r=0.25$ & $r=0.5$ & $r=0.75$ & $r=0.25$ & $r=0.50$ & $r=0.75$ \\
\hline \multicolumn{7}{|l|}{ Stable $(1.5,1.2)$} \\
\hline - rec & $0.42(0.17)$ & $0.53(0.13)$ & $0.64(0.16)$ & $0.33(0.12)$ & $0.50(0.10)$ & $0.66(0.14)$ \\
\hline - rol & $0.37(0.22)$ & $0.39(0.14)$ & $0.51(0.18)$ & $0.65(0.10)$ & $0.36(0.11)$ & $0.48(0.18)$ \\
\hline - seq & $0.81(0.08)$ & $0.79(0.09)$ & $0.81(0.04)$ & $0.76(0.14)$ & $0.70(0.13)$ & $0.80(0.05)$ \\
\hline \multicolumn{7}{|l|}{ Stable $(1.2,1.5)$} \\
\hline - rec & $0.62(0.11)$ & $0.55(0.15)$ & $0.48(0.17)$ & $0.48(0.13)$ & $0.48(0.08)$ & $0.48(0.16)$ \\
\hline - rol & $0.68(0.17)$ & $0.81(0.14)$ & $0.86(0.20)$ & $0.72(0.17)$ & $0.84(0.11)$ & $0.95(0.09)$ \\
\hline - seq & $0.83(0.03)$ & $0.83(0.05)$ & $0.84(0.01)$ & $0.83(0.02)$ & $0.82(0.03)$ & $0.82(0.03)$ \\
\hline \multicolumn{7}{|l|}{ Student $(4,2)$} \\
\hline - rec & $0.40(0.17)$ & $0.53(0.13)$ & $0.67(0.15)$ & $0.33(0.13)$ & $0.51(0.10)$ & $0.70(0.11)$ \\
\hline - rol & $0.37(0.22)$ & $0.39(0.14)$ & $0.49(0.17)$ & $0.26(0.10)$ & $0.37(0.11)$ & $0.49(0.18)$ \\
\hline - seq & $0.80(0.09)$ & $0.78(0.10)$ & $0.81(0.04)$ & $0.78(0.11)$ & $0.71(0.13)$ & $0.80(0.04)$ \\
\hline \multicolumn{7}{|l|}{ Student $(2,4)$} \\
\hline - rec & $0.58(0.20)$ & $0.42(0.22)$ & $(0.17)$ & $0.53(0.11)$ & $0.51(0.12)$ & $0.39(0.15)$ \\
\hline - rol & $0.71(0.18)$ & $0.81(0.14)$ & $0.85(0.20)$ & $0.72(0.17)$ & $0.83(0.11)$ & $0.95(0.08)$ \\
\hline - seq & $0.58(0.20)$ & $0.42(0.22)$ & $0.41(0.17)$ & $0.53(0.11)$ & $0.51(0.12)$ & $0.39(0.15)$ \\
\hline \multicolumn{7}{|l|}{$\operatorname{Burr}(4,2)$} \\
\hline \multicolumn{7}{|l|}{$\rho=-1$} \\
\hline - rec & $0.38(0.16)$ & $0.51(0.16)$ & $0.61(0.19)$ & $0.30(0.09)$ & $0.50(0.08)$ & $0.70(0.10)$ \\
\hline - rol & $0.28(0.13)$ & $0.37(0.13)$ & $0.48(0.18)$ & $0.26(0.10)$ & $0.37(0.11)$ & $0.48(0.18)$ \\
\hline - seq & $0.73(0.17)$ & $0.74(0.12)$ & $0.81(0.05)$ & $0.63(0.22)$ & $0.66(0.15)$ & $0.79(0.06)$ \\
\hline \multicolumn{7}{|l|}{$\operatorname{Burr}(2,4)$} \\
\hline \multicolumn{7}{|l|}{$\rho=-1$} \\
\hline - rec & $0.62(0.16)$ & $0.47(0.22)$ & $0.40(0.19)$ & $0.65(0.16)$ & $0.31(0.19)$ & $0.37(0.14)$ \\
\hline - rol & $0.71(0.18)$ & $0.82(0.12)$ & $0.92(0.12)$ & $0.71(0.17)$ & $0.83(0.11)$ & $0.94(0.10)$ \\
\hline - seq & $0.82(0.04)$ & $0.82(0.04)$ & $0.69(0.16)$ & $0.67(0.17)$ & $0.70(0.14)$ & $0.75(0.12)$ \\
\hline \multicolumn{7}{|l|}{$\operatorname{SVSTU}(4,2)$} \\
\hline \multicolumn{7}{|l|}{$\theta=0.95$} \\
\hline - rec & $0.40(0.17)$ & $0.52(0.14)$ & $0.64(0.17)$ & $0.35(0.14)$ & $0.51(011)$ & $0.56(0.17)$ \\
\hline - rol & $0.33(0.19)$ & $0.40(0.14)$ & $0.49(0.19)$ & $0.26(0.10)$ & $0.37(0.11)$ & $0.48(0.17)$ \\
\hline - seq & $0.77(0.12)$ & $0.75(0.11)$ & $0.80(0.06)$ & $0.78(0.10)$ & $0.70(0.13)$ & $0.79(0.06)$ \\
\hline \multicolumn{7}{|l|}{$\operatorname{SVSTU}(2,4)$} \\
\hline \multicolumn{7}{|l|}{$\theta=0.95$} \\
\hline - rec & $0.63(0.16)$ & $0.43(0.21)$ & $0.46(0.19)$ & $0.58(0.14)$ & $0.58(0.13)$ & $0.58(0.17)$ \\
\hline - rol & $0.72(0.18)$ & $0.79(0.15)$ & $0.87(0.18)$ & $0.70(0.17)$ & $0.83(0.11)$ & $0.95(0.08)$ \\
\hline - seq & $0.81(0.04)$ & $0.82(0.04)$ & $0.75(0.11)$ & $0.83(0.02)$ & $0.84(0.01)$ & $0.82(0.03)$ \\
\hline \multicolumn{7}{|l|}{$\operatorname{ARCH}(4,2)$} \\
\hline - rec & $0.41(0.18)$ & $0.52(0.15)$ & $0.65(0.19)$ & $0.37(0.15)$ & $0.60(0.14)$ & $0.75(0.09)$ \\
\hline - rol & $0.49(0.27)$ & $0.49(0.23)$ & $0.54(0.21)$ & $0.31(0.17)$ & $0.39(0.15)$ & $0.49(0.19)$ \\
\hline - seq & $0.71(0.19)$ & $0.73(0.14)$ & $0.78(0.10)$ & $0.77(0.13)$ & $0.73(0.12)$ & $0.81(0.05)$ \\
\hline \multicolumn{7}{|l|}{$\operatorname{ARCH}(2,4)$} \\
\hline - rec & $0.35(0.23)$ & $0.71(0.20)$ & $0.67(0.21)$ & $0.71(0.15)$ & $0.82(0.05)$ & $0.82(0.06)$ \\
\hline - rol & $0.70(0.20)$ & $0.70(0.21)$ & $0.62(0.27)$ & $0.71(0.17)$ & $0.78(0.16)$ & $0.88(0.19)$ \\
\hline - seq & $0.77(0.17)$ & $0.64(0.24)$ & $0.63(0.18)$ & $0.84(0.07)$ & $0.54(0.26)$ & $0.61(0.12)$ \\
\hline
\end{tabular}

Note: estimated break dates are reported for different sample sizes $(n=500,2000)$, different locations of the (true) breakpoints $(\mathrm{r}=0.25,0.50,0.75)$ and different jump scenarios $\left(\alpha_{1}, \alpha_{2}\right)$ for the tail index. "Candidate" break dates are calculated over 20,000 Monte Carlo replications. Average break date estimates are obtained by averaging over the statistically significant "candidate" breaks using the finite sample critical values. The parameters $\alpha$ and $\rho=-\beta / \alpha$ refer to the tail index and the second order parameter, respectively. The volatility persistence parameter in stochastic volatility models with student-t innovations (SVSTU) is denoted by $\theta$. 
TABLE 7. Forward and backward recursive testing outcomes

\begin{tabular}{|c|c|c|c|c|c|c|c|c|}
\hline Assets & $m^{*}$ & \multicolumn{2}{|c|}{ rec.test } & \multicolumn{2}{|c|}{ Crit.values } & \multicolumn{3}{|c|}{ Tail index and VaR (\%) } \\
\hline & & $Q_{F}$ & $Q_{B}$ & 0.95 & 0.99 & $\widehat{q}(\widehat{\alpha})$ & $\widehat{q}_{1}\left(\widehat{\alpha}_{1}\right)$ & $\widehat{q}_{2}\left(\widehat{\alpha}_{2}\right)$ \\
\hline \multicolumn{9}{|c|}{ Panel A: stock markets (local currency) } \\
\hline US & 150 & $\begin{array}{c}5.22^{* * *} \\
(\mathbf{7} / \mathbf{9} / \mathbf{8 7})\end{array}$ & 0.63 & 2.78 & 3.81 & $11.38(3.00)$ & $5.25(4.61)$ & $15.10(2.72)$ \\
\hline UK & 166 & 0.52 & 0.73 & 2.45 & 3.43 & $10.98(3.19)$ & & \\
\hline FR & 142 & 1.21 & 1.27 & 3.44 & 4.71 & $10.45(3.65)$ & & \\
\hline GE & 100 & 1.19 & 0.46 & 2.38 & 3.75 & $10.16(3.49)$ & & \\
\hline JP & 106 & 1.28 & 0.22 & 2.49 & 4.13 & $10.81(3.43)$ & & \\
\hline INDO & 128 & 1.06 & 0.96 & 3.10 & 4.49 & $23.49(2.71)$ & & \\
\hline MAL & 105 & 0.41 & 0.66 & 2.87 & 3.95 & $17.16(2.74)$ & & \\
\hline THAI & 193 & 1.33 & 0.49 & 2.84 & 3.93 & $19.19(3.06)$ & & \\
\hline MEX & 80 & 1.69 & 2.12 & 4.03 & 5.36 & $9.24(4.57)$ & & \\
\hline CHIL & 96 & 0.17 & 0.36 & 2.13 & 3.28 & $8.56(3.40)$ & & \\
\hline \multicolumn{9}{|c|}{ Panel B: bond markets (local currency) } \\
\hline US & 129 & 0.48 & 0.60 & 3.19 & 4.15 & $3.60(4.33)$ & & \\
\hline UK & 350 & 1.46 & $\begin{array}{r}7.44^{* * *} \\
(\mathbf{2 0} / \mathbf{1 0} / \mathbf{8 7})\end{array}$ & 2.96 & 4.06 & $6.44(2.57)$ & $17.52(1.89)$ & $4.23(3.03)$ \\
\hline FR & 110 & 0.52 & 0.39 & 2.94 & 4.02 & $2.82(3.94)$ & & \\
\hline GE & 81 & 1.34 & 0.95 & 2.81 & 4.26 & $2.25(4.70)$ & & \\
\hline JP & 166 & 1.81 & 2.56 & 3.20 & 4.33 & $4.79(2.58)$ & & \\
\hline \multicolumn{9}{|c|}{ Panel C: Currencies (w.r.t. US\$) } \\
\hline GBP & 119 & 1.20 & 1.18 & 2.08 & 3.20 & $5.19(3.44)$ & & \\
\hline EUR & 148 & 1.65 & 0.41 & 2.41 & 3.43 & $4.27(4.25)$ & & \\
\hline CAN & 174 & 0.61 & 0.14 & 3.29 & 4.47 & $3.98(3.33)$ & & \\
\hline JPY & 155 & 1.43 & 0.37 & 3.02 & 4.08 & $4.27(4.38)$ & & \\
\hline $\mathrm{CHF}$ & 311 & 2.86 & 1.70 & 3.80 & 5.01 & $5.61(3.72)$ & & \\
\hline MXN & 250 & 2.53 & $\begin{array}{c}6.24^{* * *} \\
(\mathbf{9} / \mathbf{1 0} / \mathbf{9 8})\end{array}$ & 2.57 & 4.010 & $30.28(1.71)$ & $280.9(1.07)$ & $11.09(2.31)$ \\
\hline CLP & 147 & 0.75 & 0.85 & 3.72 & 5.08 & $6.70(2.94)$ & & \\
\hline IDR & 267 & $\begin{array}{c}6.89 \\
\mathbf{( 2 4 / 4 / 9 8 )}\end{array}$ & $\begin{array}{c}8.2 \\
(\mathbf{3} / \mathbf{2} / \mathbf{9 9})\end{array}$ & 3.29 & 4.38 & $89.41(1.40)$ & $216.96(0.58)$ & $26.60(1.84)$ \\
\hline MYR & 51 & $\begin{array}{c}5.97^{* *} \\
(\mathbf{2} / \mathbf{1} / \mathbf{9 8})\end{array}$ & $\begin{array}{l}11.16^{* * *} \\
(\mathbf{9} / \mathbf{9} / \mathbf{9 9})\end{array}$ & 4.31 & 6.21 & $12.09(2.16)$ & $5.16(4.51)$ & $2.21(3.73)$ \\
\hline THB & 100 & $\begin{array}{c}6.48^{* * *} \\
(\mathbf{1 5} / \mathbf{5} / 97)\end{array}$ & $\begin{array}{c}7.09^{* * * *} \\
(\mathbf{1 9} / \mathbf{5} / \mathbf{9 8})\end{array}$ & 2.25 & 3.96 & $19.55(1.72)$ & $0.88(3.42)$ & $6.07(2.58)$ \\
\hline \multicolumn{9}{|c|}{ Panel D: Commodities } \\
\hline Gold & 203 & 1.92 & 2.01 & 2.44 & 3.53 & $17.36(2.74)$ & & \\
\hline Silver & 307 & 2.47 & 2.20 & 2.61 & 3.81 & $36.69(2.35)$ & & \\
\hline Oil & 150 & 1.23 & 0.96 & 3.45 & 4.56 & $23.41(3.17)$ & & \\
\hline
\end{tabular}

Note: country and currency abbreviations stand for: US (United States), UK (United Kingdom), FR (France), GE (Germany), JP (Japan), INDO (Indonesia), MAL (Malaysia), THAI (Thailand), MEX (Mexico), CHIL (Chile), GBP (British Pound), EUR (Euro), CAN (Canadian Dollar), JPY (Japanese Yen), CHF (Swiss Franc), MXN (Mexican Peso), CLP (Chilean Peso), IDR (Indonesian rupiah), MYR (Malaysian ringgit), THB (Thai Baht). The forward and backward version of the recursive test are denoted by $Q_{F}$ and $Q_{B}$, respectively. Critical values are based on 10,000 bootstrapped sample replications. Statistically significant rejections of the null hypothesis of tail index constancy at the $5 \%$ and $1 \%$ significance level are denoted by ${ }^{* *}$ and ${ }^{* * *}$, respectively. The break dates $(\mathrm{dd} / \mathrm{mm} / \mathrm{yy})$ of corresponding significant breaks are reported in bold. In case of significant breaks in the tail index, Value-at-Risk (VaR) estimates are calculated for the full sample and the subsamples determined by the break. 